\title{
Prediction and Anticipation Features- Based Intellectual Assistant in Location-Based Services
}

\author{
Ajay Kr. Gupta, MMM University of Technology, Gorakhpur, India \\ (iD https://orcid.org/0000-0001-9666-5047 \\ Udai Shanker, MMM University of Technology, Gorakhpur, India \\ (iD https://orcid.org/0000-0002-4083-7046
}

\begin{abstract}
An assistive healthcare system implementation known as IntelliAssistant has been proposed in this paper for helping the cognitively disabled peoples of mild to moderate condition by alerting them for a right traveling direction well in advance. The proposed system is based on a fuzzy inferencebased map-matching method integrated with route recommendation. Also, it uses the frequent trajectories mining method known as clustering with sequential pattern and mining for extracting data and movement prediction of a cognitive disabled person to find out the travel route to them as per their preferences. For the simulation of the proposed system, a client-server queuing model has been used. The results show the minimization of the overhead with better precision as compared to earlier already existing route recommendation systems.
\end{abstract}

\section{KEYWORDS}

Applications and Services, Location-Based Services, Mobile Computing and Networking, Speculative Computation, Trajectory Data Mining

\section{INTRODUCTION}

The use of various Intelligent Internet of Things (IoT) system's techniques (Gupta and Shanker, 2020) for big data analytics is becoming popular now-a-days in most of the industrial and domestic applications, which is attributed to high performance and very low cost for different devices. Today, rapid developments in wearable sensors, actuators, the IoT, and intelligent algorithms form the foundation for new healthcare applications including pattern recognition or prognostic modeling (Gupta and Shanker, 2020). In the immediate future, aged persons and people with disabilities can utilize smart assistive technologies to enable them to carry out day to day regular activities, enjoy the fun events, experience entertainment, and make them socialize while retaining good health and wellbeing. The function of health-related activities (DariaBattini et al., 2013), that require information and communication technologies (Elharakanyrt al., 2018) complements those smart environments. The approaches for good health and well-being make use of sophisticated networks and IoT technologies. Intellectual disabilities, also known as mental retardation or general learning disability, are a group of disorders defined by diminished cognitive and adaptive functioning. Cognitive disabilities are typically categorezed in four phases, i.e., mild (about $80 \%$ ), moderate (about 14\%), severe (about 
4\%) and extreme (about 2\%). Most cases are linked to mild to moderate type disorder. Thereofre, IntelliAssistant is a form of orientation (Liu et al., 2009; Carmien et al., 2005) for people of mild to moderate handicaps in earlier phases of cognitive disabilities (Dawe, 2006, pp. 1143-1152; American Psychiatric Association, 2013). The individual cannot use a mobile device in an advanced, serious, and extreme phase's condition, and thus the orientation is not feasible. The key features of this type of location -based services (LBS) (Gupta and Shanker, 2018) were defined as being an on-going work (Ramos et al., 2013; Sadilek\&Kautz, 2010). Augmented reality(AR) userinterfaces in LBS has been used here to guide cognitively disabled persons. It reduced the cognition effort needed to understand the route to follow. While the device determines the best travel path, a screen should appear to remind the user to wait. The traveling route (Spichkova, 2016) needs to be fed to the speculative computing module for anticipating the users' errors and ensuring the proper travel direction. The implementation and evaluation of the trajectory data mining method have been done for the aim of achieving the travel route to the user preferences. A person with cognitive impairments has the Trajectory (Lou et al., 2009) denoted as T from their $\log \mathrm{L}$. It is a sequenced series of GPS points with a successive interval that does not reach a fixed threshold $\mathrm{T}_{\mathrm{h}}$. It is represented by $\mathrm{T}: \mathrm{p}_{1} \rightarrow \mathrm{p}_{2} \rightarrow \cdots \rightarrow \mathrm{p}_{\mathrm{m}}$, where, $T_{h}>p_{i+t} \cdot t>0$ with $(m>i \geq 1)$ and $p_{i} \in P \subset L$. The $|T|$ is the number of samplings $(|T|=m)$, and $t$ is defined as the interval ofthe sampling point. $\mathrm{P}=\mathrm{p}_{1}, \mathrm{p}_{2} \cdots, \mathrm{p}_{\mathrm{m}}$ are the arrangement of points known as GPS $\log$, where each point $\mathrm{p}_{\mathrm{i}} \in \mathrm{P}$ contains $\mathrm{p}_{\mathrm{i}}$. lat, $\mathrm{p}_{\mathrm{i}} \cdot \operatorname{lng}, \mathrm{p}_{\mathrm{i}} . \mathrm{t}$ as latitude, longitude, and timestamp, respectively. Path $\mathrm{W}$ is a related street fragment arrangement that starts at vertex $\mathrm{V}_{\mathrm{i}}$ and ends at $\mathrm{V}_{\mathrm{j}}$, i.e., $\mathrm{W}: \mathrm{e}_{1} \rightarrow \mathrm{e}_{2} \rightarrow \cdots \rightarrow \mathrm{e}_{\mathrm{n}}$, where, $\mathrm{V}_{\mathrm{i}}=\mathrm{e}_{1}$. start, $\mathrm{V}_{\mathrm{j}=} \mathrm{e}_{\mathrm{n}}$. end, $\mathrm{e}_{\mathrm{k}+1}$. start $=\mathrm{e}_{\mathrm{k}}$.end, $1 \leq \mathrm{k} \leq \mathrm{n}$. A summary of the GPS client movement $\log \&$ Trajectory is given in Figure 1.

ESRI built the ArcGIS in 1999, which is a geographic information system method as its name goes written in the $\mathrm{C}++$ programming language. It essentially helps us to record, archive, evaluate, process, and handle enormous volumes of mapped data on the LBS platform. Suppose, an individual is traveling down a finite street road, N, which we don't know. Instead, we have an ArcGIS map framework with road network $\mathrm{G}(\mathrm{V}, \mathrm{E})$. For simplicity, a one to one correspondence is assumed between arcs from $\mathrm{G}$ and roads from N. G(V, E) consists of dimensional arcs reflecting roads as shown in Figure 2. The finite sequence of values for labeled points $\left(A_{0}, A_{1}, \ldots, A_{n}\right)$ will define increasing arc, $A \in E$. These are the junctions or dead ends of an arc used for the switching to another arc. In the GPS, $\mathrm{P}_{\mathrm{t}}$ denotes the actual position of the individual at time $\mathrm{t}$, and $\mathrm{P}_{\mathrm{et}}$ is the approximate location at this moment.

One can receive route information (Bajracharya, 2016) by periodically collecting data from the GPS over a server connected with a mobile phone. Recalculations of repeated visit locations can produce greater precision at the expense of considerable resources (Gupta and Shanker, 2018). It is important to incorporate a dynamic location management algorithm with continuous monitoring of GPS request to fix the user position, to define a route for the user and to utilize the resources efficiently (Gupta and Shanker, 2018). Apart from main users, another person known as caretaker might need to run the application. Thus, the system has a localization function to let the caretaker know the current position of the cognitive disability person in real-time. This function is very much useful because the caretaker may create a different activity after knowing a person's location. The proposed InelliAssistant could be added with more features through some external systems such as

Figure 1. Log and trajectory for moving person

\begin{tabular}{|cccc|}
\hline & Latitude & Longitude & Time \\
& & & \\
$\mathbf{p}_{1}$ & $28.7041^{\circ}$ & $77.1025^{\circ}$ & $22: 31$ \\
$\mathbf{p}_{2}$ & $25.4223^{\circ}$ & $79.5467^{\circ}$ & 22.33 \\
$\ldots \ldots \ldots \ldots$ & $\ldots \ldots \ldots$. & $\ldots \ldots \ldots$ & $\ldots \ldots \ldots .$. \\
$\mathbf{p}_{n}$ & $30.2234^{\circ}$ & $83.3435^{\circ}$ & 22.43 \\
\hline
\end{tabular}

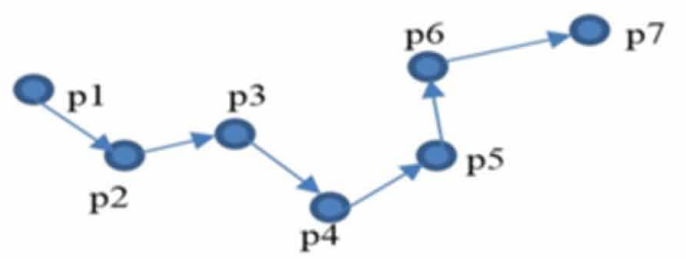




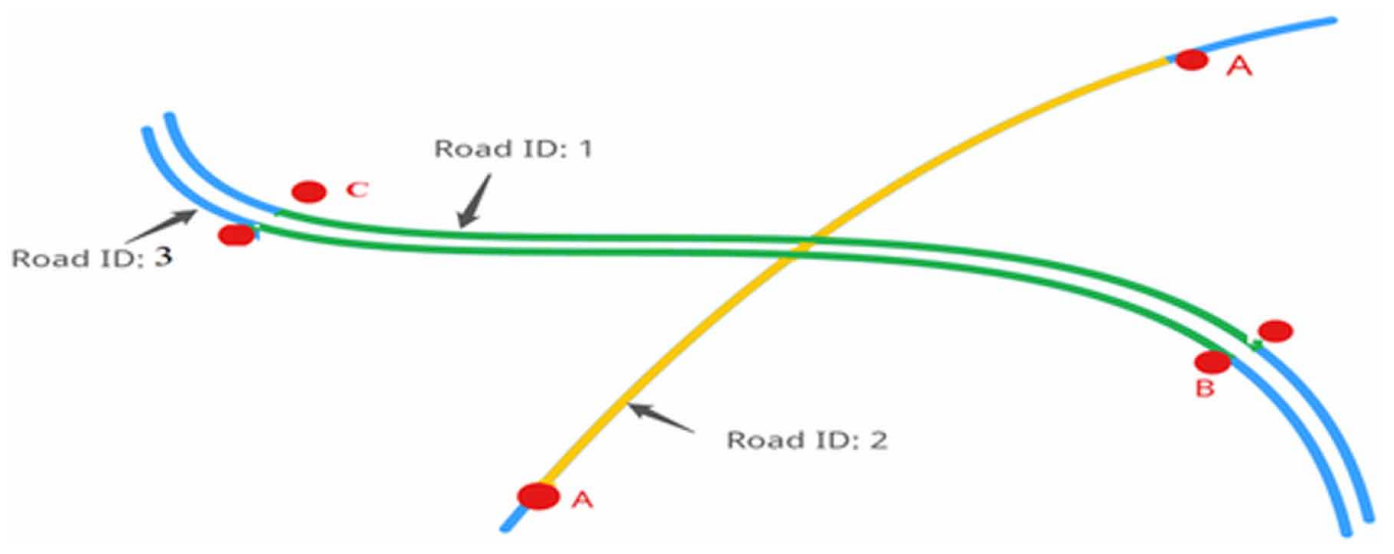

Ambient Intelligence (AmI). With this added functionality, the instruction process can be initiated remotely, whenever the consumer has an appointment to ensure timely arrival. AmI makes research attraction in evolving science and technology areas relating to an IoT system's capacity to perceive the surrounding environmental conditions and reacts to people's presence, expanding on humancentered computing, context awareness, profiling, ubiquitous computing, and pervasive computing.

The proposed LBS (IntelliAssistant) has a predictive computing feature to direct the user with users' error predicting and alerting capabilities. The aim of the research work here is to suggest a framework for integrating software as a service for disabled or older people with reduced cognitive abilities by providing access to easy and intuitive programs that are personalized to each person's requirements. The proposed solution helps to improve the user's independence and well-being in the home or outdoor surroundings and to reduces his isolation by allowing the user to contact other people easily. Previously defined alternatives show lack of orientation and did not involve a module for tracking cognitively disabled persons while s/he is going outside. To build applications considering persons with cognitive disabilities, it is important to understand the model functionality to optimize its accessibility and usability. The user's physical and/or cognitive deficiencies may be reduced due to the minimization of executive function in the LBS application. IntelliAssistant can be described in a composed module of Map-Matching (MM) with a low sampling rate (Lou et al., 2009)and GPS route recommendation (Spichkova, 2016).

The aim of the MM module in the IntelliAssistant is an exploration of the path corresponding to $T$ from Graph $G$ by matching the actual position $P_{t}$ to the approximate location $P_{e}$ with its true direction. The chain of user locations identified is linked on a virtual map by generating a spatial map of roads from the traces. For every LBS MM, it is a preprocessing phase. Because of two reasons given ahead, the need for an efficient MM algorithm is significant. The primary reason is errors in GPS systems, which lead to a person's wrong position. The secondary reason is the low sampling rate, bandwidth limitation, and storage cost (Gupta and Shanker, 2021) which lead to hindrance in route recommendation procedure. It is also a critical activity to monitor the data processing for traffic monitoring and recommending routes for a moving person on the map. If the LBS MM imprecision is largeer because of device fault or poor bandwidth, the system should be able to find the moving person accurately on the map. So, LBS with the capability of continuous monitoring of the moving person, GPS position request rate, and moving person route creation needs is to be introduced. To minimize MM error, the sensor data is translated through the specific MM algorithms e.g. Fuzzy Logic, Dempster method, Bayesian inference, Kalman Filter, Particle Swarm Optimization (PSO) and Shafer's theory of increasing the accuracy that is accessible at all times (Gupta and Shanker, 2020a) (Gupta and Shanker, 2020b). The previous policies simply disregard the error factors associated with 
the system of a road map and even the orientation method as well. Focused on these limitations, this paper argues multi-criteria fuzzy logic-based MM model and Sequential Pattern Mining \& Clustering (SPMC) based route recommendation (Gupta and Shanker, 2020a) to be used in the proposed IntelliAssistant. Apart from this, one may also collect data from stake holders such as road constructors, road users, and traffic officers. Using these data, MM framework androad network components (Kizito and Semwanga, 2020) can be integrated to develop a qualitative modeled system. This system would help in possibly reducing the road accident incidences. The main contributions of the paper are outlined with given remarks.

1. Fuzzy Inference System (FIS) Integrated Navigation System- FIS uses various input parameters e.g. GPS set interval time, horizontal precision dilution, the number of epochs, velocity direction, heading error, heading shift, location-based perpendicular length from edge of the candidate, etc. to integrate an accurate MM algorithm in IntelliAssistant.

2. Evaluation of False Matching Relationships with Traditional MM Method- This paper contains a descriptive analysis of the suggested FIS MM approach to be used in IntelliAssistant. The Fuzzy Logic dependent algorithm shows better MM performance in terms of accuracy and recall and more resistant to sampling interval variance compared to those of traditional MM algorithms.

3. Use of Sequential Pattern Mining \& Clustering Algorithm for Route Recommendation- SPMC is used for the mining of frequent mobility patterns (FMPs) and framing of mobility rules (R) to be used in route recommendation.

4. Self-trained Augmented Reality (AR) Supporting Path Recommendation Framework- User movement trajectory is fed up to the self-trained augmented reality framework to anticipate user movement errors. This helps in the correct traveling direction guidance and cognitive effort reduction required for understanding the path to follow.

5. A survey of broad varieties of compatible algorithms to resolve the issue of MM and recommendation has been given.

The remainings of paper are written as follows. The related works linked to this research are given in Section 2. In Section 3, the Fuzzy based MM algorithm, sequential pattern mining, and clustering for route guidance are presented. Also, we explain simulation results with a comparative study in section 4 . Section 5 gives the conclusion and discusses the aforementioned future scope of the work in the current research domain.

\section{RELATED WORKS}

Several researchers made an in-depth study and work on MM methods. MM methods do the job of locating a moving car on a road utilizing map data in digital form with GPS receiver knowledge. MM implementations serve many functions such as travel time estimation (Tang et al., 2018; Rahmani et al., 2017), route selection parameter estimation (Oyama and Hato, 2018), analysis of gridlock network (Oyama and Hato, 2017), and motion anomaly identification (Qin et al., 2019). In India, we claim our cities are being transformed into a smart cities; therefore, we are in the need of a major GIS network to turn a simple city into a smart one. Beyond that, we need to know about technical knowledge of QGIS, R, PostGIS, and SQL, etc. which are the free frameworks with excellent functionality in GIS for Feature-based MM having Trajectories for GPS with Low Sampling Rate (Yin et al. 2018).

MM methods based on the probabilistic strategy (Yin et al., 2016) are built to deal with complex road or intersections segments, which apply comprehensive calculation and greatly enhance matching precision. A probabilistic approach based MM includes multiple hypotheses (Yuan et al., 2010), and Hidden Markov Model (HMM)-dependent MM for GPS positioning (Ren and Karimi, 2009). These approaches rely on the view of the overall circumstance for all candidate road links, and all location 
details (Qinglin et al., 2015), rather than the estimation of specific positions and neighboring candidate road links. The positioning data sampling size often affects the precision of the positioning data $\mathrm{Hu}$ et al., 2009) (Gupta and Shanker, 2021). Mapping for cell phone positioning and small sample rate GPS mapping need incremental gains in terms of precision and reliability. Several sophisticated maps matching algorithms utilizing Hidden Markov Models (HMM) (Mohamed et al., 2017; Yang and Gidófalvi, 2018), fuzzy logic model (Quddus et al. 2006), Kalman filtering (Cho and Choi, 2014), etc. have been developed to solve complex road markings. Usually, HMM is used to define the optimal matching route while addressing the noise and sparse position details.

The trajectories include inconsistencies related to acceleration, traveling speed, predicted distance between points, changes in direction, etc. Therefore, before the map generation process, a filtering stage is needed, in which traces are checked for any irregularities. Typically, all such approaches takes 3 steps. The initial step is to extract the direct link with soft constraints close to the distance to the positioning point. This process guarantees true trajectories selection having strong degree of duplicity. A practicality attribute to every link is assigned in the next step. The accuracy relies on topological MM practicability attributes, such as the connectivity between topologies, the intersection of angles, the similarity of directions, and projective deviations. The third stage is to measure all the ties accrued at each period.

Optimization is one of the most common approaches in the early phase of this field of research. In the area of robot mapping, MM is a significant and difficult issue of object identification. Georgiou et al. (Georgiou et al., 2017) reported that the current system implementation requires enhancement in the efficacy of robot-human cooperation by measuring the maps correspondence of real map to the map built by sensors of a robot. In a topological map, path matching is a similar subject, which can then be used to align maps (Schwertfeger and Yu, 2016). Zhou et al. (Zhou et al., 2020) and tackle the problem of map exploring robotic systems by applying a collaborative algorithm organizing an autonomous mobile robot team to navigate an unfamiliar area. Schuessler et al. (Schuessler et al., 2009) had provided computational algorithms focused on the methodology of multiple hypotheses. Statistic first picks all nodes inside the system defined radius across the sampling point for one unmatched trajectory. After that, it attaches all paths in the region that link to the candidate road collection with at least one of those chosen nodes. The corresponding score is determined and recorded in the current route candidate chart by allocating a path sampling point to each road candidate. The corresponding score is determined by integrating the sampling point heading concerning the free-flow speed on the route, path heading, and the existing sampling point speed. When the current sampling point has been processed for all candidates route, this method assigns the path to become the matched route which achieved the maximum score. However, if only a few candidate roads are available, then it will expand the radius repeatedly until sufficient candidates are present for each stage of sampling.

In (Lou et al., 2009), ST MM is implemented with the speed (i.e., temporal) and geometric (i.e., spatial) constraints to overcome the small-sampling GPS point problem. The algorithm ST-MM could be divided into two processes, namely filtering of the candidate, and ST Analysis. The ST-MM measures the weight feature for two successive GPS sampling locations and the candidate points thereof. The weight feature that the ST-MM considers is focused solely on the temporal and spatial constraints. It considered internal variables, (radius of error circle and count of candidate points) within the road network to identify the most possible segments of candidate roads. By pursuing the highest summation of weight points, the ST-MM produces a real direction. ST-MM algorithm work in the assumption that a moving person will always take the shortest path which is the case not conform to the real scenario. In the observational evaluation, we find that the weight method in the ST-MM technique uses the shortest route queries (SPQ) amongst two successive GPS points. This is the most costly aspect of the MM method that reduces the answer time and the QoS of the total matching process. A significant number of SPQs are performed in the MM technique, owing to a set number of candidate points (CP) and error circle radius (ECR). The GridST (Chandio et al., 2017) attempts to enhance the management of the first step, i.e. filtering of candidate step. The radius of the error 
circle and also the actual number of applicant points chosen are dynamically modified as per the geography of the road system. The amount of shortest path calculations is consequently decreased, thereby, reducing the total run time complexity. GridST breaks the road system diagram into grids to create the locality of the road system. Before the actual MM procedure, the knowledge of all grids is measured and arranged to ensure better run time complexity of the method. When a grid seems to have a larger volume of road segments, there would be larger possibilities for candidate extraction to pick more amount of candidate points in a smaller error area.

Based on the used trajectories range, current MM techniques may be classified into two categories i.e. local/incremental MM method (Liang et al., 2016), and global MM method (Liu et al., 2017). The first category is based on a greedy approach that uses the current trajectory point to decide the initial point in MM, which is followed by the subsequent points. It applies navigation with turn-byturn in real-time applications. The local/incremental method has better accuracy with a higher rate of sampling.However, when the rate of sampling declines, the issue of arc-skipping (Lou et al., 2009) relates to the situation arises. In this scenario, the car switches straight from one roadway fragment to another when it is already far from the junction, gets ridiculous, and creates a major drop in precision. The local/incremental method would also have a fairly large energy demand because of the high errate sampling.

Some new approaches have been developed to enhance matching precision, such as weightbased MM (Sharath et al., 2019), spatial-temporal feature-based mapping (Hsueh and Chen, 2018), and topology mapping (Wang et al., 2017). Even so, these methods are not completely efficient, and still, scope exists for further research development. A global mapping method is attemped to locate a curve well into the network of roads that is just as near to the vehicle path as possible. It involves a full collection of track points before the discovery of the most common trajectory path on the road network. The correlations between several line segments are calculated using Frechet distance (Wei et al. 2013), using long common subsequence (LCS) (Zhu et al ., 2017), or using probability function (Millard-Ball et al., 2019; Knapen et al ., 2018; Rappos et al., 2018) in global matching approaches.

Millard-Ball et al. (Millard-Ball et al., 2019) used a three-segment quasi-probability equation, which consists of temporal probability, topological probability, and geometric probability to attain effective candidate range match. Knapen et al. (Knapen et al . 2018) tried to arrange them chronologically GPS traces. They evaluated the highest probability of partial routes depending on a directed acyclic graph. Besides, Rappos et al. (Rappos et al ., 2018) suggested a force-directed MM system focused on the distance, GPS points to road edge angle, and length. Yang and Gidófalvi (Yang and Gidófalvi, 2018) described a rapid MM technique combining precomputation with Markov hidden model. Algizawy et al. (Algizawy et al., 2017) strengthen the HMM used in MM to facilitate it with an adaptive probability for highly sparse smartphone data.

To produce correct mapping results within a fairly short period, AntMapper-matching (Gong et al., 2017) using Ant colony synthesis method has been discussed for local and global geometric/ topological information. Due to the scarcity of more information, the precision of Global algorithms increased; however, they also suffer from long computation high processing time. Therefore, this paper tried to reduce the high computation and processing time of used FIS-MM in Intelliassistant. The Intelliassistant's MM technique increases the matching quality of high-frequency data and utilizes the junction segment matching approach to increase the matching precision for trajectory data in an urban environment having ahigher rate of sampling (Akin and Alasalvar, 2016).

\section{MM AND TRAJECTORY PATTERN MINING ALGORITHMS}

In case of an individual being the first time user of this application, the system will not have any user details. In this case, when there is information about the consumer beforehand, the program determines the shortest path using GraphHopper to retrieve the information. Having predictions regarding object movement from observational points requires to have a mechanism for this movement, for which 


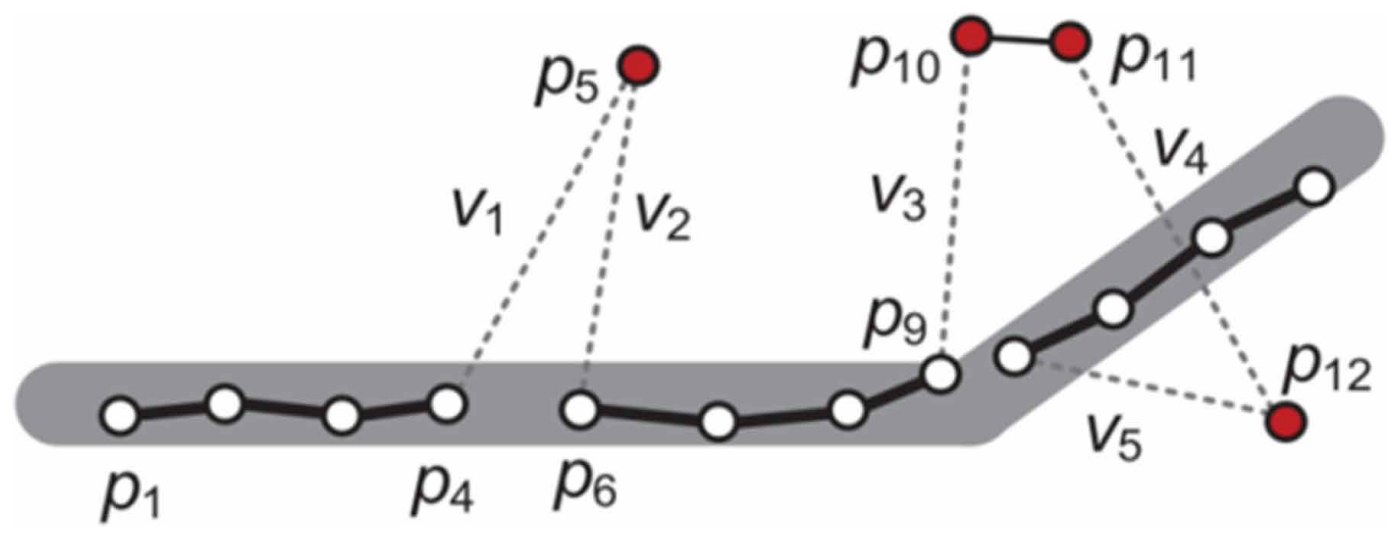

a huge collection of spatio-temporal (Gupta and Shanker, 2020) options are available as given in the related work section. One of its easiest and most commonly implemented methods is the linear regression, which works in the assumption of constant movement direction and constant velocity between each pair of successive observations. A preprocessing phase is required to adjust the path to use as raw data for the extraction process. The random movements not matching to other patterns do not contribute to the mining result and are treated as outliers in the preprocessed trajectory data. Some of the patterns in trajectories have an error greater than the normal GPS error (where the given GPS observation is more than 50 meters away from a median point between pair of previous and successive GPS observations) which is treated as noise. Due to sensor noise and other causes, such as receiving weak positioning signals in a low bandwidth environment, spatial trajectories are rarely entirely correct. In cases, as seen in Figure 3 , a noise point error like $\mathrm{p}_{4}$ is too high to extract valuable knowledge, for example, the speed of travel.

Therefore, before beginning a mining process, we must remove these noise points from trajectories. This stage is called the preprocessing of trajectories and is a basic aspect of several tasks in trajectory mining. Removing the noise of the results, deletion of points that may not increase trajectory information, and matching the map are important. The purpose of noise filtering is to eliminate any noise points from a trajectory that could be induced by the weak GPS signal. Although, the noise reduction issue has not been completely solved, some of the common methods for eliminating trajectory noise are Mean (or Median) Filter, Heuristics-based Outlier Detection, Kalman and Particle Filters.

The method for the mining of trajectories is shown in Figure 4. For the first step of preprocessing, LBS has used an open-source tool GraphHopper Directions API (Karich\& Schroder, 2017), which has a corresponding map feature. The next step is the MM procedure which is the operation of acquiring the remainder of the information and matching geographical coordinates to the Open Street Map database. The subsequent step is the application of a frequent trajectories mining method known as SPMC for extracting the data. We are experimenting with good results for this cycle and in our preliminary tests with an SPMC system consisting of sequential patterns mining and Clustering algorithm which group clusters locations. SPMF (Fournier, 2017) is an open-source API used in the SPMC method (Gupta and Shanker, 2019). Using the SPMC algorithm, we can extract frequent trajectories from the users' past traveling trajectories. The input called support threshold of a sequential sequence is used in the SPMC method to define a minimum number of sequences required to appear as a pattern. The system uses a data mining approach with user data that will be powered by the system. Thus, the user expectations are adapted to the route according to the patterns threshold in the dataset. In the case, when the route recently created using the mined patterns is larger than the previous shortest path, the device will use this shortest path. Using the SPMC clustering module, the data is arranged into 


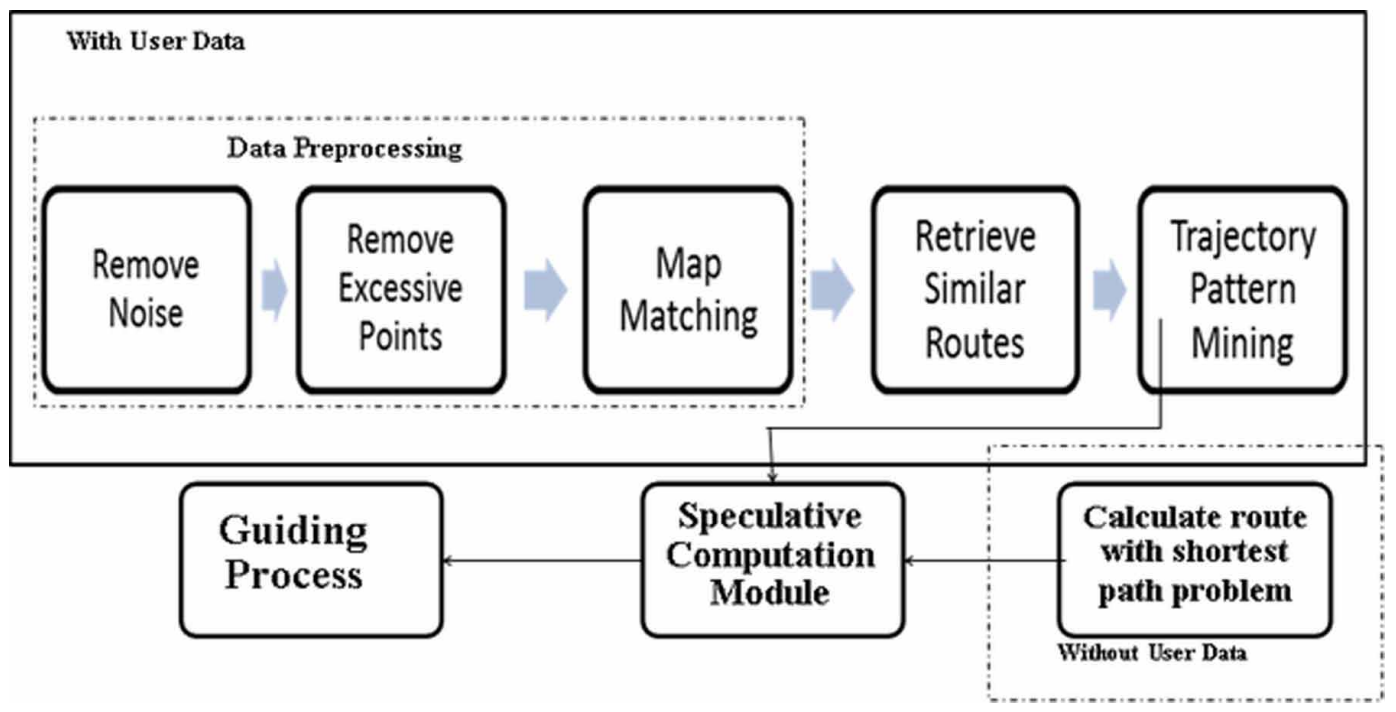

clusters taking into account the input parameters. Using the mining process, connections between the clusters must be created to obtain a route that directs the person to the intended destination from his current locations.

The MM algorithm and trajectory data mining methods to be used in Intelliassistant are described in this section. MM is the process of comparing reported geographical coordinates to actual road network functional models which make the job easier of urban computation, for example, traffic application, etc. The MM problem can be based on as described in Figure 5. In the MM algorithm, the traveling route is fed up in a speculative computation platform for predicting user mistakes and maintaining the right direction of travel.

The FIS-MM algorithm is used in the proposed framework. The implementation of this MM is based on a global MM methodology i.e. ST-MM (Lou et al., 2009). Weight function in the STMM strategy uses the shortest path queries (SPQ) which is the most costly aspect of MM operation between two successive GPS points. SPQ computation reduces the response time and QoS of overall matching. A large number of SPQs are executed because of the MM approach fixed count constraints of candidate points and error circle radius. The compulsion of minimum amount of interior variables (candidate points and error circle radius) in MM may result in poor accuracy when the corresponding portion of a road system does not have a sufficient count of candidate points.

A comprehensive experiment finding has been given in subsequent sections that offer insight into the pros and cons of the new MM method that allows participants to incorporate the right algorithm for LBS. Modern map details integration in MMGPS reading of odometer $(\Delta d)$, gyro rate reading $(\Delta \theta)$, and associated errors $\left(\varepsilon_{\theta}\right)$ are responsible for the necessary performance of navigation. Zhao and his colleagues (Zhao et al., 2014) use the EKF algorithm for this combination of GPS and DR. The key requirement in any $\mathrm{MM}$ algorithm is to find the actual position of any moving individual on a route. GPS/DR outputs are the velocity of the person (v), Easting $(E)$, Northing $(N)$, heading $(\theta)$, and variances of error (i.e., $\sigma_{v}, \sigma_{e}, \sigma_{n}, \sigma_{h}$ ). MM provides the correct road link as the output with their trust value greater than the defined threshold. If, no link is available in the candidate location area, the program assumes that the car is off the road from known avenue ties. The selection strategy would be easy in a situation where the applicant field contains just one segment. If there are several links along with fixed positions, an approximation method such as FIS could be used to find the correct hyperlink between the candidate ties. Any model for matching maps is composed of two parts. The 

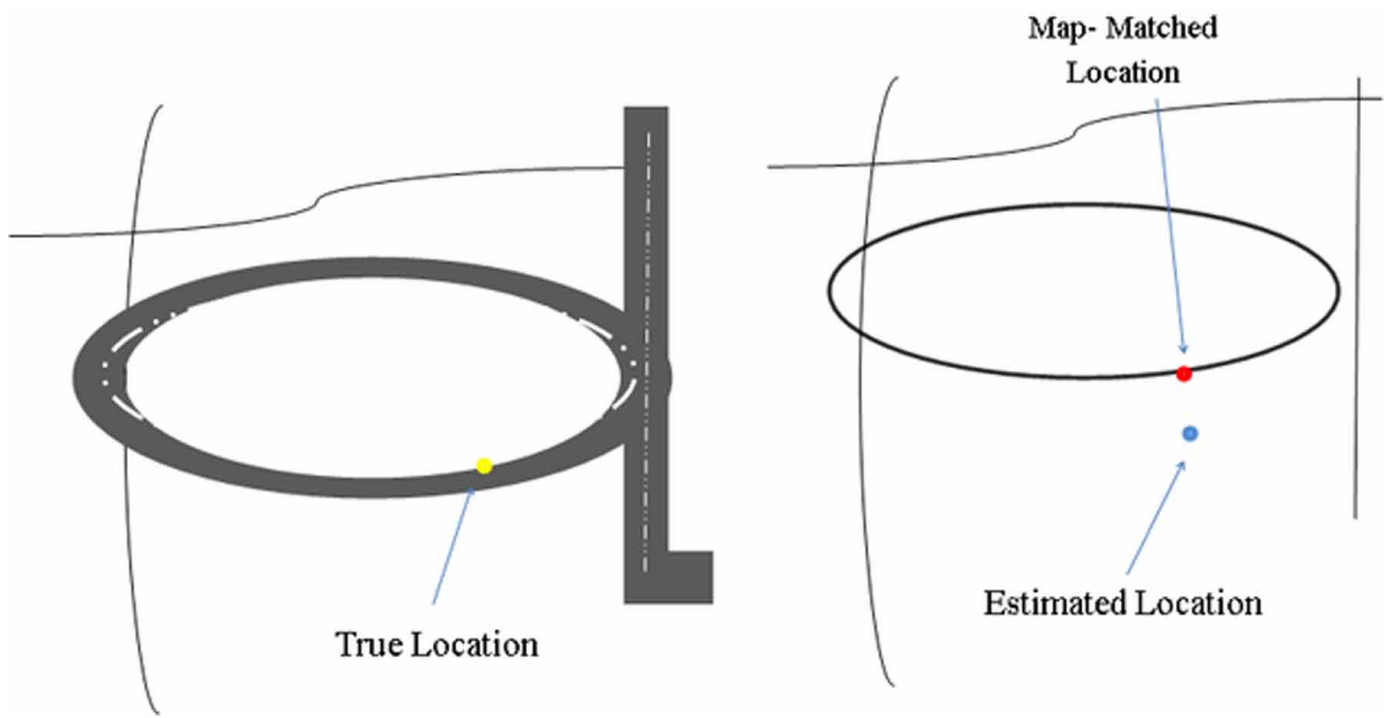

first section has the steps for proper recognition of the link and the second section has steps for position determination on the link chosen. The initial MM method is the location fix feature to the perpendicular distance of the path, the path bearing, and the orientation of the individual and the first fixed time of the GPS receiver. The search space is extracted from the variance of error. First link identification is done through a few fixes of the first link position.

\subsection{Fuzzy Inference System Based MM}

In Matlab 6.5, the Adaptive Neural Fuzzy Inference System (ANFIS) can be used to refine fuzzy membership features. The selection of membership functions is achieved through a system of at least squares or backpropagation. The if-then rules allow the precedent (input) for the corresponding (output) relation in the FIS. Based on their criticality, the weights in FIS are established. The practical fuzzy reasoning comprises certain significant steps that are shown in Figure 6.

FIS can be based on Mamdani (i.e. the output of weighted expression) or Sugeno (i.e. the output of the fuzzy set). Mamdani's most fundamental difference from Sugeno FIS is how crisp output is produced from fuzzy data. For the defuzzification method, Mamdani uses the Center of Gravity technique; whereas Sugeno FIS applies a weighted average function to measure the crisp output. The Mamdani FIS is more popular mainly because it offers rational outcomes with a fairly easy layout, and also because of the rule base's intuitive and interpretable existence. Mamdani FIS can be used specifically with both MIMO (multiple inputs multiple outputs) and MISO (multiple input single output) systems while the SUGENO FIS can only be used in MISO systems. MISO deals with the structures of the same linguistic performance vector (i.e. weighted average output) while MIMO deals with the framework where the outputs of the laws are generally independent of each other. Aggregating various nature outputs with a weighted average of MM approaches don't make logical sense. Here, we considered Mamdani FIS since it captures the highest performance through max-min function. In a fuzzy scheme, the collection of suitable membership functions shapes, and their parameters depend on the question that one has to solve. Though, membership function (MF), that takes values between 0 and 1 , requires any of them to never hit 1 . It can be useful to reflect the knowledge that is never certain. Likewise, if one doesn't have an aprioriin the form of MFs, triangular or trapezoidal shapes are simple to enforce and fast to compute. However, if one has any a priori on their shapes 
Figure 6. FIS for IntelliAssistant implementation

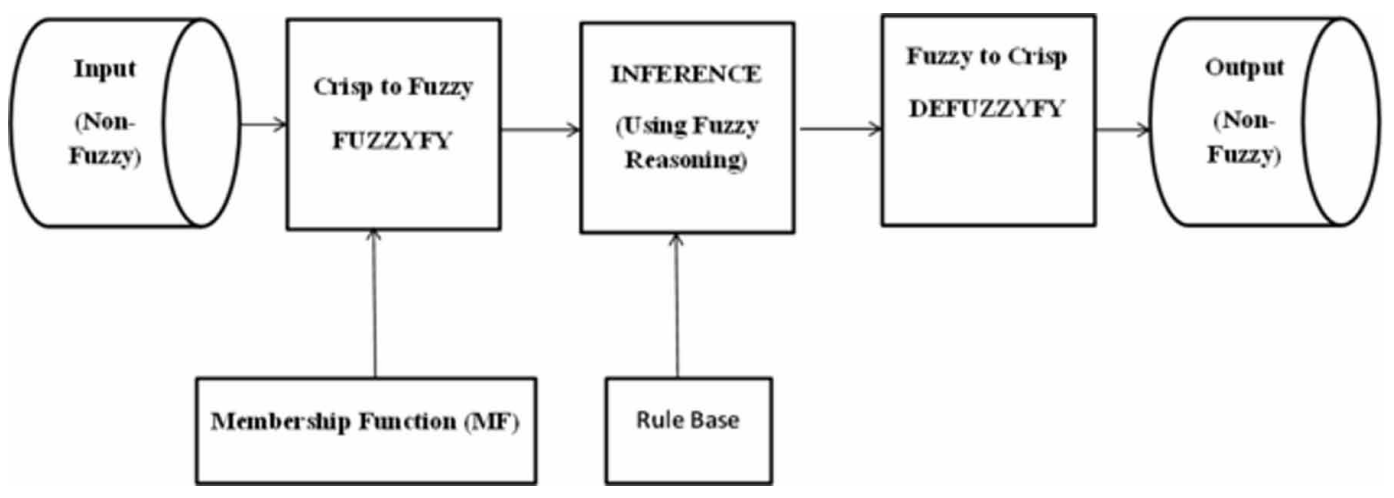

(e.g., sampled data or histograms), it might be useful to create MFs with shapes extracted from such a priori shapes, if possible after some smoothing.

To pick the best MFs, the training results are compared using MAPE (Mean Average Percentage Error) to equate the outcome with the real one. The MF outcome comparison is conducted with the actual one dependent on percentage error and the best MF having the lowest percentage error are selected in the proposed system.

To apply the Fuzzy logic, we have used FIS based on Mamdani. It fuzzifies the parameter value in the range of $[0,1]$. Various forms of membership feature can be used but for the convenience of the interface, we choose the simplified form functions to define membership. Triangular membership function (TMF) is a commonly used and simple function to define membership. For every parameter $\left(\mathrm{Pt}_{\mathrm{i}}\right)$ from parameter set (PS), FIS fuzzifiesits corresponding value $\mathrm{v}_{\mathrm{i}}$ in the range [0, 1] from value set $v$ which lies between $[a, b]$. The number of boundaries selection should be done with the consideration of precision and running complexity trade-off. The TMF i.e. $\mu \mathrm{f}(\mathrm{x})$ can be represented mathematically as given by equation 1 .

Membership Function $(\mu \mathrm{f})=\left\{\begin{array}{cc}0, & b \leq x \leq a \\ \frac{b-x}{c-b}, & a \leq x \leq \operatorname{cor} c \leq 0 \leq b \\ 0, & b \leq x\end{array}\right.$

The parameters $\mathrm{a}$ and $\mathrm{b}$ are two boundaries that must be tailored and standardized for each boundary condition. Parameter $\mathrm{c}$ is the point at which membership function has its highest value. The representation of the TMF is given in Figure 7.

Membership function $\mu \mathrm{f}(\mathrm{x})$ mapping value $\mathrm{v}_{\mathrm{i}} \in \mathrm{v}$ range is in between 0 and 1 from value set which lies between $[\mathrm{a}, \mathrm{b}]$. This procedure of mapping is called parameter fuzzification. If a function has $n$ boundaries condition, then the $2 n$ boundary parameters need to be optimized. More formally, if $\mathrm{Pt}_{\mathrm{i}} \in \mathrm{PS}$ has $\mathrm{b}_{\mathrm{i}}$ boundaries, then count of used optimization variables for generating FIS output in a fuzzy form in a limit of maximum and minimum values is given by equation 2 .

Count of Optimization Variables $=2 * \sum_{i=1}^{n} b_{i}+2$ 
Figure 7. Graphical overview of the triangular membership function

\section{$\mu \mathbf{f}(\mathbf{x})$}

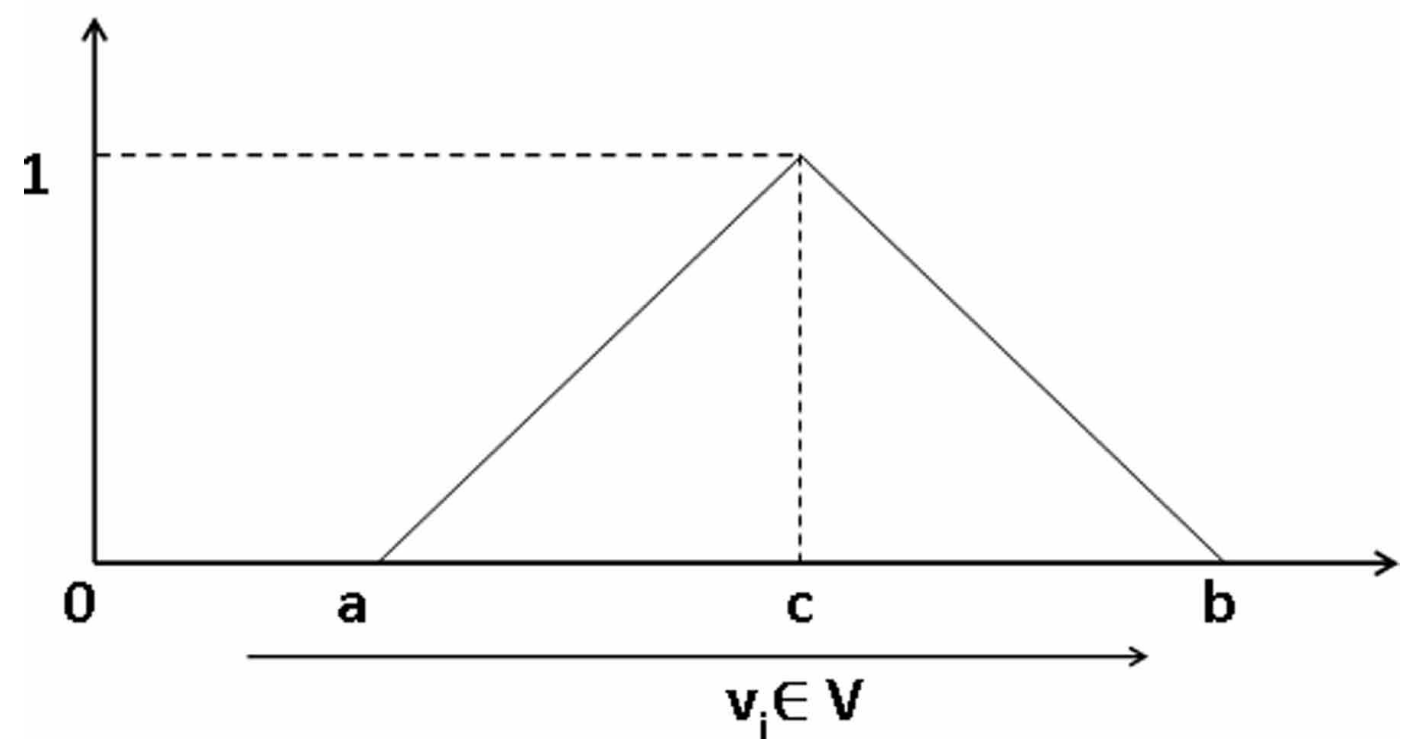

The weighted average procedure is used for crisp output computation of associated likelihood for a link. For each fuzzy rule, the degree of applicability computation is obtained by $\min ()$ function. The following listed terms are extracted from $a$ to $b$ for measuring probability-value, provided a certain range of membership functions as given in equation $3,4,5,6$.

$$
P t_{f 1}=\frac{v_{1}-a}{b-a} P t_{f 2}=\frac{v_{2}-a}{b-a} \ldots \ldots . P t_{f n}=\frac{v_{n}-a}{b-a}
$$

Range $\left(\mathrm{Pt}_{\mathrm{f} 1}, \mathrm{Pt}_{\mathrm{f} 2}, \mathrm{Pt}_{\mathrm{f} 3}\right) \rightarrow[0,1]$

$\mathrm{Pt}_{\min }=\min \left(\mathrm{Pt}_{\mathrm{f} 1}, \mathrm{Pt}_{\mathrm{f} 2}, \mathrm{Pt}_{\mathrm{f} 3}\right)$

Likelihood-value $=10 \times\left(1-\frac{\int_{a}^{b}\left[P t_{\text {min }} \times\left(P t_{\text {min }} \times(b-a)+a\right)\right] d x}{\int_{a}^{b}\left[P t_{\text {min }} d x\right.}\right)$

$\mathrm{Pt}_{\mathrm{fn}}$ reflects the fuzzified value of some parameter $\mathrm{Pt}_{\mathrm{n}} \cdot \mathrm{Pt}_{\text {min }}$ is the minimum value after fuzzification for all parameters. Fuzzy logic MM algorithm uses a collection of input variables e.g. amount of epochs, GPS fix period (obtained from GPS), heading fault, HE (degree), Heading shift (obtained from the gyro) (HI), perpendicular distance of location production from the applicant component PD 


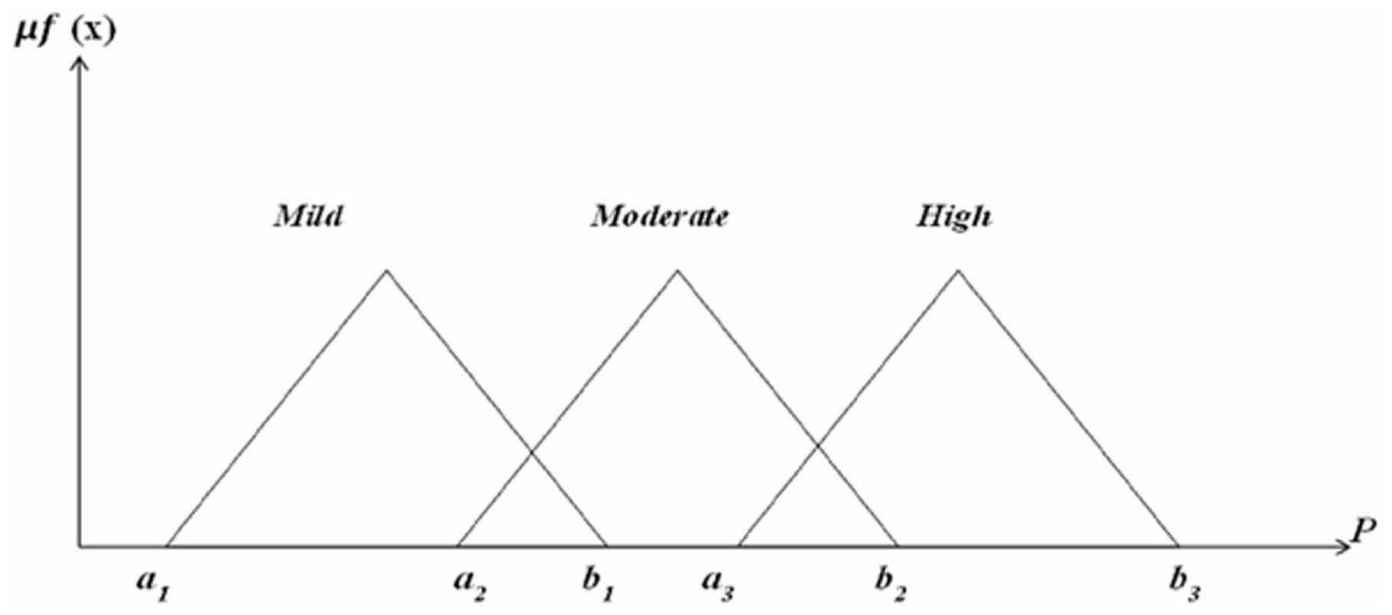

(m), HDOP (Horizontal Dilution of precision), movement direction w.r.t. links, velocity direction w.r.t. links, and moving object velocity $\mathrm{v}(\mathrm{m} / \mathrm{sec})$. The fuzzy logic $\mathrm{MM}$ algorithm implements three forms of Fuzzy rule sets. First fuzzy rule sets is used for source link, second set of fuzzy rule is used for person location on this link, and third fuzzy rule sets is used for the link junctions to find successive link.

Likelihood-value dependence on $v_{i}$ is presumed during rule base creation, which is calculated by moving person simulation. For increasing parameter, restricted boundary numbers such as Mild, Moderate, and High are defined based on that parameter's fuzzified value as shown in Figure 8. Let $S_{F}=\{$ Mild, Moderate, High $\}$ where $S_{1}[i]$ is the $i^{\text {th }}$ element of fuzzy set $S_{F}$ for first parameter vector. Similarly, $S_{n}[i]$ refers toith ${ }^{\text {th }}$ value of the $n^{\text {th }}$ parameter for $i \leftarrow 1,2,3 \ldots \ldots$ etc. Below is the general category of rules in the rule base, where $S_{1}, S_{2}$, and $S_{3}$ denote a limit set for the three consecutive parameters. The FIS MM output is computed probability for fixing of the location on the link. In the suzeno fuzzy map, constants that fit are taken to be 10 as Mild, 50 as Moderate, and 100 as high. The rules for Fuzzy logic control are set out in Table 1.

Initial good fix as indicated in Figure 9 is denoted by P. FIS tests edge 1 as the true link for location fix P. The FIS then considers the edge 1 as a true link for location fix $\mathrm{Q}$ and $\mathrm{R}$ in the corresponding phase. Thus, it can be found that the location fix $S$ is present on the edge 1 .

\subsection{SPMC Based Next Location Estimation}

The SPMC consists of a procedure to mine frequent mobility. Let $\mathrm{P}_{\mathrm{k}-1}=\left\langle\left(\mathrm{l}_{1}, \mathrm{t}_{1}\right),\left(\mathrm{l}_{2}, \mathrm{t}_{2}\right) \ldots\left(\mathrm{l}_{\mathrm{k}-1}, \mathrm{t}_{\mathrm{k}-1}\right)\right\rangle$ is the frequent (k-1)-pattern and $\mathrm{V}\left(\mathrm{l}_{\mathrm{k}-1}\right)$ is the coverage region Cell $1_{\mathrm{k}-1}$ neighbor set of cells. The pattern mining involves attaching $p=\left(v, t_{\mathrm{k}}\right)$ at the end to generate a candidate $k$-pattern. Here, $\mathrm{t}_{\mathrm{k}-1}$ is the timestamp of $\mathrm{l}_{\mathrm{k}-1}$ and $t_{\mathrm{k}}$ is the timestamp of $\mathrm{v}$. Then, candidate k-patterns selection is done by supp min. $_{\text {. }}$

The predicted region (Gupta and Shanker, 2017) consists of cells which are locations in the predicted region. To simulate client movement behavior and understand how frequent pattern mining is being processed here, we have used mobility log file dataset of users. A sample trajectory log file is given in Table 2. Table 3 and Table 4 are Frequent Mobility1-Patterns and Frequent Mobility2Patterns for the given trajectory. The candidate k-patterns selection from frequent mobility pattern set are achieved by Algorithm 1: Acquire FMPs (L).

The support value of $\left\langle\left(\mathrm{L}_{4}, \mathrm{t}_{2}\right)\right\rangle,\left\langle\left(\mathrm{L}_{14}, \mathrm{t}_{8}\right)\right\rangle$ is less than supp $\mathrm{min}_{\text {min }}=3$. So, they are discarded and not to be a part of the next step. 
Table 1. Rule set for IntelliAssistant MM

\begin{tabular}{|c|c|c|c|}
\hline Perpendicular Distance & $\begin{array}{l}\text { Horizontal Dilution } \\
\text { of Precision }\end{array}$ & $\begin{array}{c}\text { Gyro Processed Heading Change } \\
\text { (Hi) }\end{array}$ & $\begin{array}{l}\text { L1 (Rule-Set } \\
\text { Out) }\end{array}$ \\
\hline$M I L D$ & $M I L D$ & $M I L D$ & $M I L D$ \\
\hline$M I L D$ & $M I L D$ & MODERATE & $M I L D$ \\
\hline$M I L D$ & $M I L D$ & $H I G H$ & MODERATE \\
\hline$M I L D$ & MODERATE & $M I L D$ & $M I L D$ \\
\hline$M I L D$ & MODERATE & MODERATE & MODERATE \\
\hline$M I L D$ & MODERATE & $H I G H$ & MODERATE \\
\hline$M I L D$ & $H I G H$ & $M I L D$ & $M I L D$ \\
\hline$M I L D$ & $H I G H$ & MODERATE & $M I L D$ \\
\hline$M I L D$ & $H I G H$ & $H I G H$ & $H I G H$ \\
\hline MODERATE & $M I L D$ & $M I L D$ & $M I L D$ \\
\hline MODERATE & $M I L D$ & MODERATE & MODERATE \\
\hline MODERATE & MILD & $H I G H$ & MODERATE \\
\hline MODERATE & MODERATE & $H I G H$ & MODERATE \\
\hline MODERATE & MODERATE & MODERATE & MODERATE \\
\hline MODERATE & MODERATE & $H I G H$ & $H I G H$ \\
\hline MODERATE & $H I G H$ & $M I L D$ & $M I L D$ \\
\hline MODERATE & $H I G H$ & MODERATE & $H I G H$ \\
\hline MODERATE & $H I G H$ & $H I G H$ & $H I G H$ \\
\hline$H I G H$ & $M I L D$ & $M I L D$ & $M I L D$ \\
\hline$H I G H$ & $M I L D$ & MODERATE & $M I L D$ \\
\hline$H I G H$ & $M I L D$ & $H I G H$ & MODERATE \\
\hline$H I G H$ & MODERATE & $M I L D$ & MODERATE \\
\hline$H I G H$ & MODERATE & MODERATE & $H I G H$ \\
\hline$H I G H$ & MODERATE & $H I G H$ & $H I G H$ \\
\hline$H I G H$ & $H I G H$ & $M I L D$ & $H I G H$ \\
\hline$H I G H$ & $H I G H$ & MODERATE & $H I G H$ \\
\hline$H I G H$ & $H I G H$ & $H I G H$ & $H I G H$ \\
\hline
\end{tabular}

Algorithm A1: Acquire FMPs (L)

Input: ${ }_{1} \leftarrow$ Legth-1 large pattern set // Let Lk = Legth-K large patterns set

$C_{1}$ Legth-1 candidate patterns set // Let $C_{k}=$ Legth- $K$ candidate patterns set

$\mathrm{G}=$ Directed graph for neighboring information,

Supp $_{\text {min }}$

Output: $L=$ FMP set.

Begin

$\mathrm{k}=0$

Repeat 


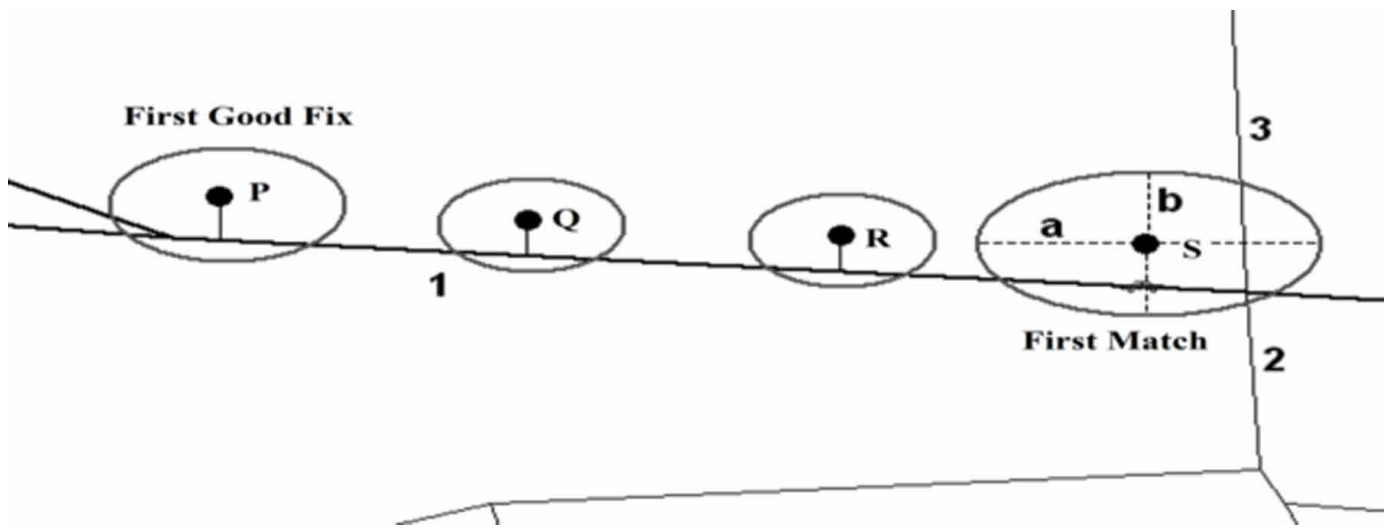

Table 2. Trajectory log file

\begin{tabular}{|c|c|}
\hline Sequence ID & Mobility Patterns \\
\hline 1. & $<\left(\mathrm{L}_{5}, \mathrm{t}_{3}\right),\left(\mathrm{L}_{1}, \mathrm{t}_{5}\right),\left(\mathrm{L}_{15}, \mathrm{t}_{7}\right)>$ \\
\hline 2. & $<\left(\mathrm{L}_{5}, \mathrm{t}_{3}\right),\left(\mathrm{L}_{1}, \mathrm{t}_{5}\right),\left(\mathrm{L}_{13}, \mathrm{t}_{9}\right)>$ \\
\hline 3. & $<\left(\mathrm{L}_{5}, \mathrm{t}_{3}\right),\left(\mathrm{L}_{2}, \mathrm{t}_{4}\right),\left(\mathrm{L}_{1}, \mathrm{t}_{5}\right),\left(\mathrm{L}_{15}, \mathrm{t}_{7}\right)>$ \\
\hline 4. & $<\left(\mathrm{L}_{5}, \mathrm{t}_{3}\right),\left(\mathrm{L}_{2}, \mathrm{t}_{4}\right),\left(\mathrm{L}_{1}, \mathrm{t}_{5}\right),\left(\mathrm{L}_{13}, \mathrm{t}_{9}\right)>$ \\
\hline 5. & $<\left(\mathrm{L}_{2}, \mathrm{t}_{4}\right),\left(\mathrm{L}_{1}, \mathrm{t}_{5}\right),\left(\mathrm{L}_{15}, \mathrm{t}_{7}\right)>$ \\
\hline 6. & $<\left(\mathrm{L}_{2}, \mathrm{t}_{4}\right),\left(\mathrm{L}_{1}, \mathrm{t}_{5}\right),\left(\mathrm{L}_{13}, \mathrm{t}_{9}\right)>$ \\
\hline 7. & $<\left(\mathrm{L}_{3}, \mathrm{t}_{2}\right),\left(\mathrm{L}_{2}, \mathrm{t}_{4}\right),\left(\mathrm{L}_{12}, \mathrm{t}_{6}\right),\left(\mathrm{L}_{13}, \mathrm{t}_{9}\right)>$ \\
\hline 8. & $<\left(\mathrm{L}_{3}, \mathrm{t}_{2}\right),\left(\mathrm{L}_{2}, \mathrm{t}_{4}\right),\left(\mathrm{L}_{12}, \mathrm{t}_{6}\right),\left(\mathrm{L}_{14}, \mathrm{t}_{8}\right)>$ \\
\hline 9. & $<\left(\mathrm{L}_{4}, \mathrm{t}_{1}\right),\left(\mathrm{L}_{3}, \mathrm{t}_{2}\right),\left(\mathrm{L}_{2}, \mathrm{t}_{4}\right),\left(\mathrm{L}_{12}, \mathrm{t}_{6}\right),\left(\mathrm{L}_{13}, \mathrm{t}_{9}\right)>$ \\
\hline 10. & $<\left(\mathrm{L}_{4}, \mathrm{t}_{1}\right),\left(\mathrm{L}_{3}, \mathrm{t}_{2}\right),\left(\mathrm{L}_{2}, \mathrm{t}_{4}\right),\left(\mathrm{L}_{12}, \mathrm{t}_{6}\right),\left(\mathrm{L}_{14}, \mathrm{t}_{8}\right)>$ \\
\hline
\end{tabular}

Table 3. for k=1, Frequent Mobility1-Patterns

\begin{tabular}{|l|l|l|l|l|}
\hline${ }_{1}$ & & & ${ }^{L} \mathbf{l}$ & \\
\hline Candidates 1-Patterns & Support & & Frequent 1-Patterns & Support \\
\hline$<\left(\mathrm{L}_{5}, \mathrm{t}_{3}\right)>$ & 4 & & $<\left(\mathrm{L}_{5}, \mathrm{t}_{3}\right)>$ & 4 \\
\hline$<\left(\mathrm{L}_{2}, \mathrm{t}_{4}\right)>$ & 8 & & $<\left(\mathrm{L}_{2}, \mathrm{t}_{4}\right)>$ & 8 \\
\hline$<\left(\mathrm{L}_{3}, \mathrm{t}_{2}\right)>$ & 4 & & $<\left(\mathrm{L}_{3}, \mathrm{t}_{2}\right)>$ & 4 \\
\hline$<\left(\mathrm{L}_{4}, \mathrm{t}_{1}\right)>$ & 2 & & $<\left(\mathrm{L}_{1}, \mathrm{t}_{5}\right)>$ & 6 \\
\hline$<\left(\mathrm{L}_{1}, \mathrm{t}_{5}\right)>$ & 6 & $<\left(\mathrm{L}_{15}, \mathrm{t}_{7}\right)>$ & 3 \\
\hline$<\left(\mathrm{L}_{15}, \mathrm{t}_{7}\right)>$ & 3 & & $<\left(\mathrm{L}_{13}, \mathrm{t}_{9}\right)>$ & 3 \\
\hline$<\left(\mathrm{L}_{13}, \mathrm{t}_{9}\right)>$ & 3 & $<\left(\mathrm{L}_{12}, \mathrm{t}_{6}\right)>$ & 3 \\
\hline$<\left(\mathrm{L}_{12}, \mathrm{t}_{6}\right)>$ & 3 & & \\
\hline$<\left(\mathrm{L}_{14}, \mathrm{t}_{8}\right)>$ & 2 & & & \\
\hline
\end{tabular}


Table 4. for k=2, Patterns of Mobility \& FMPs

\begin{tabular}{|l|l|l|l|l|}
\hline$C_{2}$ & & & L & \\
\hline Candidates 2-Patterns & Support & & Frequent 2-Patterns & Support \\
\hline$<\left(\mathrm{L}_{5}, \mathrm{t}_{3}\right),\left(\mathrm{L}_{2}, \mathrm{t}_{4}\right)>$ & 2 & & $<\left(\mathrm{L}_{2}, \mathrm{t}_{4}\right),\left(\mathrm{L}_{13}, \mathrm{t}_{9}\right)>$ & 4 \\
\hline$<\left(\mathrm{L}_{5}, \mathrm{t}_{3}\right),\left(\mathrm{L}_{1}, \mathrm{t}_{5}\right)>$ & 2 & $<\left(\mathrm{L}_{2}, \mathrm{t}_{4}\right),\left(\mathrm{L}_{12}, \mathrm{t}_{6}\right)>$ & 4 \\
\hline$<\left(\mathrm{L}_{5}, \mathrm{t}_{3}\right),\left(\mathrm{L}_{13}, \mathrm{t}_{9}\right)>$ & 2 & $<\left(\mathrm{L}_{1}, \mathrm{t}_{5}\right),\left(\mathrm{L}_{15}, \mathrm{t}_{7}\right)>$ & 3 \\
\hline$<\left(\mathrm{L}_{2}, \mathrm{t}_{4}\right),\left(\mathrm{L}_{1}, \mathrm{t}_{5}\right)>$ & 2 & $<\left(\mathrm{L}_{1}, \mathrm{t}_{5}\right),\left(\mathrm{L}_{13}, \mathrm{t}_{9}\right)>$ & 3 \\
\hline$<\left(\mathrm{L}_{2}, \mathrm{t}_{4}\right),\left(\mathrm{L}_{13}, \mathrm{t}_{9}\right)>$ & 4 & & & \\
\hline$<\left(\mathrm{L}_{2}, \mathrm{t}_{4}\right),\left(\mathrm{L}_{12}, \mathrm{t}_{6}\right)>$ & 4 & & & \\
\hline$<\left(\mathrm{L}_{3}, \mathrm{t}_{2}\right),\left(\mathrm{L}_{5}, \mathrm{t}_{3}\right)>$ & 0 & & & \\
\hline$<\left(\mathrm{L}_{3}, \mathrm{t}_{2}\right),\left(\mathrm{L}_{2}, \mathrm{t}_{4}\right)>$ & 2 & & & \\
\hline$<\left(\mathrm{L}_{1}, \mathrm{t}_{5}\right),\left(\mathrm{L}_{15}, \mathrm{t}_{7}\right)>$ & 3 & & & \\
\hline$<\left(\mathrm{L}_{1}, \mathrm{t}_{5}\right),\left(\mathrm{L}_{13}, \mathrm{t}_{9}\right)>$ & 3 & & & \\
\hline$<\left(\mathrm{L}_{15}, \mathrm{t}_{7}\right),\left(\mathrm{L}_{13}, \mathrm{t}_{9}\right)>$ & 0 & & & \\
\hline$<\left(\mathrm{L}_{12}, \mathrm{t}_{6}\right),\left(\mathrm{L}_{13}, \mathrm{t}_{9}\right)>$ & 2 & & \\
\hline
\end{tabular}

$\mathrm{k}=\mathrm{k}+1$

Foreach Legth-k-large patterns set $\mathrm{F}_{\mathrm{k}}=<\left(\mathrm{p}_{1}, \mathrm{t}_{1}\right),\left(\mathrm{p}_{2}, \mathrm{t}_{2}\right), \ldots, \quad\left(\mathrm{p}_{\mathrm{k}}\right.$, $\left.t_{k}\right)>\in L_{k}$ do

$\mathrm{N}\left(\mathrm{p}_{\mathrm{k}}\right) \leftarrow\left\{\mathrm{n} \mid \mathrm{n}\right.$ is the adjacent cell of $\left.\mathrm{p}_{\mathrm{k}-1}\right\}$

For each node $n \in N\left(l_{k-1}\right)$ do

$\mathrm{U}\left(\mathrm{p}_{\mathrm{k}}\right) \leftarrow\left\{\mathrm{s}=\left(\mathrm{n}, \mathrm{t}_{\mathrm{k}}\right) \mid \mathrm{t}_{\mathrm{k}+1}>\mathrm{t}_{\mathrm{k}}\right.$ and $\left.<\left(\mathrm{n}, \mathrm{t}_{\mathrm{k}}\right)>\in \mathrm{N}\left(\mathrm{p}_{\mathrm{k}-1}\right)\right\}$

Foreach $s=\left(n, t_{k}\right) \in U\left(p_{k}\right)$ do

$C=\left\langle\left(p_{1}, t_{1}\right),\left(p_{2}, t_{2}\right), \ldots,\left(p_{k}, t_{k}\right),\left(n, t_{k+1}\right)>\right.$

$\mathrm{C}_{\mathrm{k}+1}=\mathrm{CUC}_{\mathrm{k}+1}$

Endfor

Endfor

Endfor

For each mobility pattern $B \in D$ do

$\mathrm{F} \leftarrow\left\{\mathrm{q} \mid \mathrm{q} \in \mathrm{C}_{\mathrm{k}+1}\right.$ and $\mathrm{q}$ is a subsequence of $\left.\mathrm{B}\right\}$

For each $\mathrm{C} \in \mathrm{C}$ do

$q \cdot$ count $=q \cdot$ count +1

Endfor

Endfor

$L_{\mathrm{k}+1} \leftarrow\left\{q \mid q \in_{\mathrm{Ck}+1}\right.$ and . support $\left.\geq_{\text {supp }_{\text {min }}}\right\}$

$\mathrm{L}=\mathrm{L} \mathrm{UL}_{\mathrm{k}+1}$

While $L_{\mathrm{k}} \neq \varnothing$

Return $L$

End

\subsubsection{Framing Mobility Rules and Next Cell Prediction}

The mobility patterns rules can be defined as given in equation 7 .

$\mathrm{R}: \mathrm{X} \rightarrow \mathrm{Y}$ such that $\mathrm{X} \cap \mathrm{Y}=\varnothing$. 
where, $\mathrm{X} \& \mathrm{Y}$ are two FMPs. Let, the all FMP set is represented by $\mathrm{S}$. Then, mobility rules can be defined as given in equation 8 .

$$
\mathbf{M} \rightarrow(\mathbf{S}-\mathbf{M}) \quad \text { for } \operatorname{all}(\mathrm{M} \subset \mathrm{S}) \&(M \neq \varnothing)
$$

The value of confidence $(R)$ is associated with each rule $(R)$. The confidence $(R)$ can be computed as defined in equation 9.

Confidence $(\mathbf{R})=\frac{\operatorname{support}(\boldsymbol{A} \cup \boldsymbol{B})}{\operatorname{support}(\boldsymbol{A})} \times 100$

Confidence $(\mathrm{R})$ is the confidence threshold for rule $(\mathrm{R})$. Rules of frequent mobility filtering are attained by Confidence $(\mathrm{R})$. A temporal weighted value $\left(\mathrm{w}_{\mathrm{i}}\right)$ is associated with each rule, which is estimated by using equation 10 .

$$
\text { weight }(\boldsymbol{R})=\frac{\text { RuleTime }- \text { MinTime }}{\text { MaxTime }- \text { MinTime }} * 100
$$

where, Rule ${ }_{\text {Time }}$ is the time of rule's tail the last point. In log file, the recorded minimum and maximum time is represented by $\mathrm{Max}_{\text {Time }}$ andMin ${ }_{\text {Time }}$ respectively.

After framing of rules and associating weights, they are used further in prediction of next probable cell visit by the mobile user. The proposed mobility prediction algorithm is presented in Algorithm A2.

Algorithm A2: Next Cell_Prediction ( $R, P)$

Input:Rules Set $=\mathrm{R}$

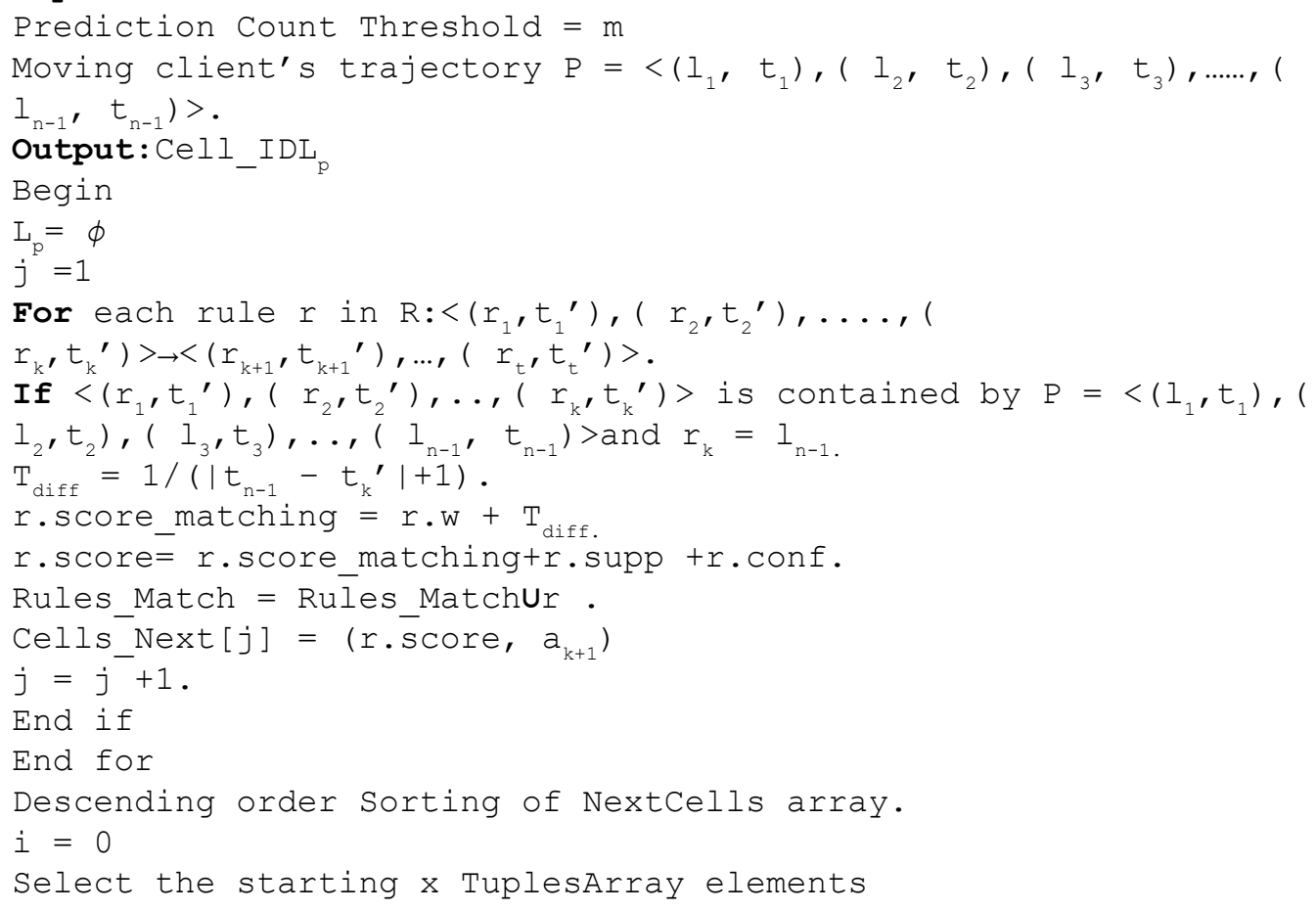


While ( $i<x$ and $i<$ TuppleArray.length) do

$L_{p}{ }^{-} L_{p}$ UTuppleArray [i]

Cell_ID $=L_{p}$

ReturnCell_ID

End

\section{SIMULATION RESULTS OF THE INTELLIASSISTANT WITH EXAMPLE DATASET}

Microsoft Visual Studio 2005 (C++) with ArcGIS is used here for the implementation of algorithms on a computer with 32 GB RAM, i7-4770 K CPU, Intel Core, and $3.50 \mathrm{GHz}$. Information on the map is processed with R-tree spatial indexing (Wolfson, Sistla, Chamberlain \&Yesha 1999). The implementation uses Microsoft Research Asia public GPS Geolife dataset to deploy the coverage area in SPMC. This data collection comprises of 182 people, 18,670 trajectories, with a cumulative distance of approximately 1200,000 kilometers and cumulative period 48,000 + hours. The GPS points in trajectory is a triplet containing three parts with longitude, latitude, and timestamp. We took 3465 Trajectory dataset sequences with a random selection of the start and end location and a given individual speed range in the simulator being implemented. We have set a total duration of 10 places and an average length of 15 places visited from the trajectory dataset. Shorter segments are likely to yield unreliable results as the traces of GPS tend to be noisier immediately after a capturing gap. Therefore, they are generally neglected. In our study, the PC with the required configuration took approximately 18 minutes to a maximum of 254 minutes in framing the mobility rules using clustered sequential pattern mining. Using the A* algorithm (Hart, Nilsson \& Raphael, 1968), the FIS dependent MM techniques discovered the shortest path. Two evaluations are performed in the study. First, the estimation of results MM (precision and effectiveness) are evaluated from real driving knowledge, whose direction was already established. The second assessed the obtained precision improvement after parameters trained in the experimentation. For algorithm execution, the system needs a navigation device comprises of a 16-bit 80C196 KB microcontroller, user interface device, positioning system, and path guidance system. The speed range for the traveling human is between $1 \mathrm{~km} / \mathrm{h}$ and $5 \mathrm{~km} / \mathrm{h}$ (Liu et al., 2009; Carmien et al., 2005; Ramos et al., 2013). Table 5 represents the quantity of data surrounding the road map and the number of nodes. FIS MM uses a confusion matrices as outlined in Table 6.

In this matrix representation, $\mathrm{T}_{1}$ and $\mathrm{T}_{2}$ reflect the amounts that the given approach results correctly accept in the true match and amount that the given approach correctly rejects the true mismatches respectively. $F_{1}$ and $F_{2}$ are the numbers incorrectly arising from the specified approach as a true mismatch in an accurate match and a true match to accurate mismatch respectively. Some of the most common metric schemes are provided by scientists to calculate the efficiency of the algorithm such as accuracy, recall, and precision. The following formulas as given by equation 11, 12,13,14, and 15 are used for evaluating the corresponding findings in this report.

Table 5. Road Map Data and Nodes

\begin{tabular}{|l|l|l|l|}
\hline \multicolumn{1}{|c|}{ Area } & \multicolumn{1}{|c|}{ Application } & \multicolumn{1}{c|}{ Road Count } & \multicolumn{1}{c|}{ Count of Nodes } \\
\hline \multirow{2}{*}{ Delhi } & Source data & 72 & 60 \\
\cline { 2 - 4 } & Target data & 64 & 54 \\
\hline
\end{tabular}


Table 6. Confusion Matrix in MM

\begin{tabular}{|c|c|c|c|}
\hline \multirow[t]{4}{*}{ Computed } & \multicolumn{3}{|l|}{ Actual } \\
\hline & & Match & Mismatch \\
\hline & Matches & $\mathrm{T}_{1}$ & $F_{1}$ \\
\hline & Mismatch & $\mathbf{F}_{2}$ & $\mathbf{T}_{2}$ \\
\hline
\end{tabular}

Accuracy $=\frac{T_{1}+T_{2}}{T_{1}+T_{2}+F_{1}+F_{2}}$

Positive Recall $=\frac{T_{1}}{T_{1}+F_{2}}$

Negative Recall $=\frac{T_{2}}{T_{2}+F_{1}}$

Positive Precision $=\frac{T_{1}}{F_{1}+F_{2}}$

Negative Precision $=\frac{T_{2}}{T_{2}+F_{2}}$

Accuracy and effectiveness are used to evaluate the navigation system performance. The recall is the ratio of the results that were accurately observed by algorithm, while precision refers to the ratio of results that were truly estimated i.e. true match and true mismatch regardless of whether these measurements areaccurateor not. The results of the experiments are given in Table 7. It shows that due to algorithm parallelism, fuzzy logic dependent MM algorithm's reciprocal precision is improved from 51 percent to 92 percent. However, the proposed algorithm's recall rate has risen dramatically from 60 to 94 percent. Equation 16 can be used for F_Measure, which is a function of Recall and Precision.

$$
\mathrm{F}_{-} \text {Measure }=2 * \frac{\text { Precision } \times \text { Recall }}{\text { Precision }+ \text { Recall }}
$$

Running time and accuracy in this work were the key evaluation parameter for online MM techniques. Many scientists have proposed strategies for enhancing efficiencies. Figure 10 shows the various methods for the sampling intervals. The count of trajectories used for the analysis is 
Table 7. Comparative study of traditional \& fuzzy dependent MM algorithm used in IntelliAssistant

\begin{tabular}{|c|c|c|c|c|}
\hline Method & Recall & Precision & F-Measure & Accuracy \\
\hline Fuzzy based MM & 94 & 90 & 92 & 91 \\
\hline Traditional MM & 60 & 51 & 55 & 56 \\
\hline
\end{tabular}

Figure 10. Running time evaluation of different MM algorithm used in IntelliAssistant

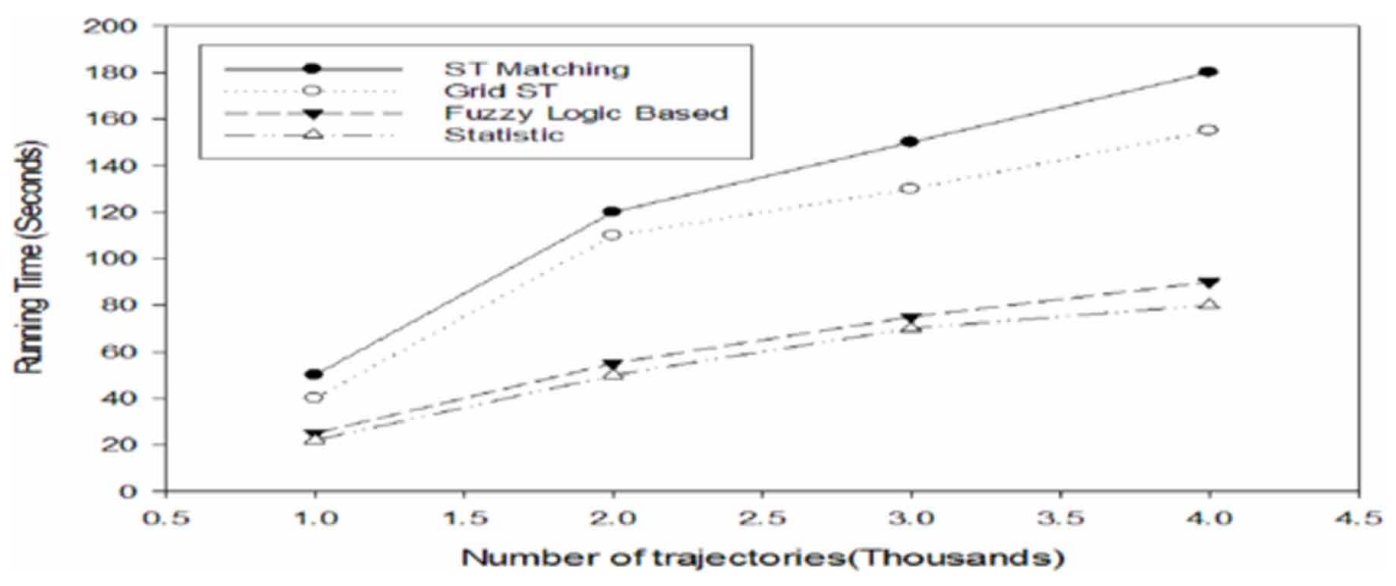

represented by the horizontal axis and the vertical axis describing running time. A comparison of all four algorithms based on runtime performance is given in Table 8 .

We compared the 3 algorithms GridST (Chandio, Tziritas, Zhang \& Xu, 2015), Statistics (Schuessler, Axhausen \& Zurich, 2009) and ST-Matching (Lou et al., 2009) with proposed MM algorithm used in IntelliAssistant. Runtime efficiency of GridST, Statistics indicates, ST-Matching and fuzzy logic based MM are proportional to the amount of navigation device trajectories. ST-Matching and GridST algorithms require a time-consuming matrix measurement and they lag the running time complexity of the FuzzyLogic method with its statistics.

Figure 11 depicts the proposed model implementation precision with variation in sampling periods. The vertical axis indicates precision, whereas, the horizontal axis represents the sampling time. The four algorithms are compared based on accuracy output as given by Table 9 .

Simulation results show that the evaluated four algorithms have identical patterns in matching precision. The FIS MM technique is more reliable than the techniques used earlier. GridST and STMatching algorithms have identical matching precision, due to similar basic logic. Statistics MM

Table 8. Effects of trajectories count on conventional \& fuzzy logic-based MM algorithm in Intelliassistant

\begin{tabular}{|c|c|c|c|c|}
\hline & \multicolumn{2}{|c|}{ Running Time of MMMethods (In Seconds) } & ST MM & $\begin{array}{c}\text { Fuzzy Logic } \\
\text { MM }\end{array}$ \\
\hline $\begin{array}{c}\text { Trajectory count (In } \\
\text { Thousands) }\end{array}$ & Statistic & Grid ST & 50 & 25 \\
\hline 1 & 22 & 40 & 120 & 55 \\
\hline 2 & 50 & 110 & 150 & 75 \\
\hline 3 & 70 & 130 & 180 & 90 \\
\hline 4 & 80 & 155 & & \\
\hline
\end{tabular}


Figure 11. Evaluation of the effect on accuracy by the sampling rate for the different MM algorithm used in IntelliAssistant

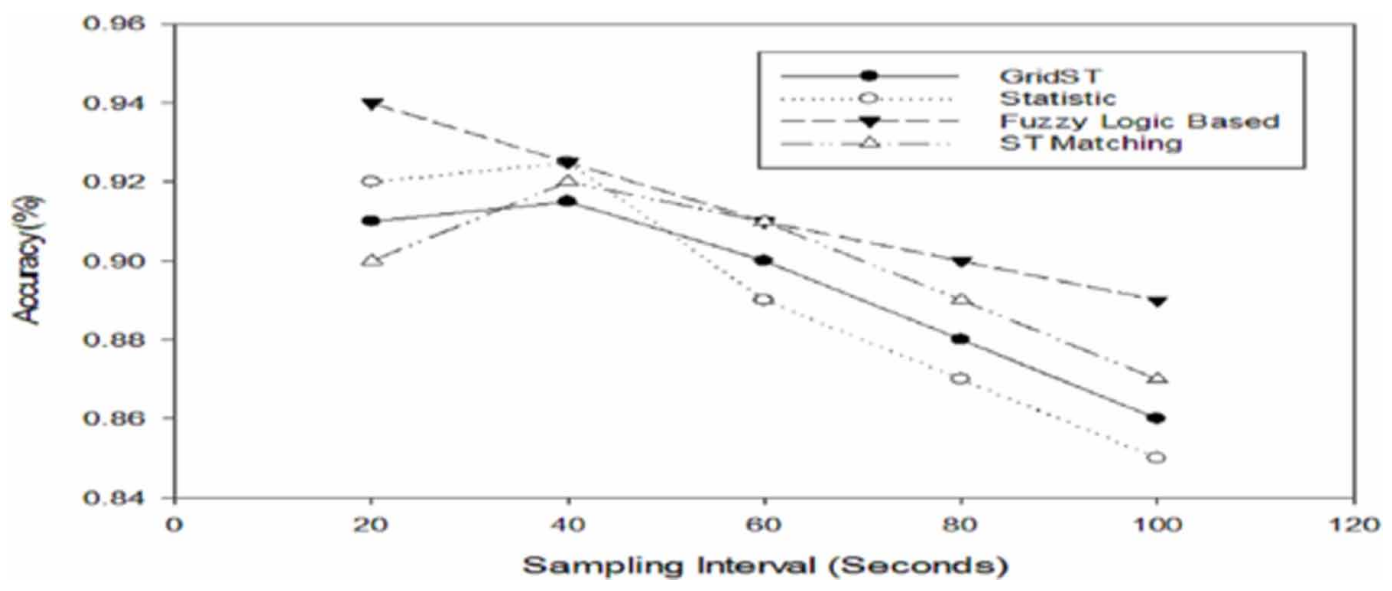

Table 9. Impact of variation in intervalof sampling on accuracy of conventional MM and Intelliassistant's FIS-MM

\begin{tabular}{|c|c|c|c|c|}
\hline & \multicolumn{4}{|c|}{ Accuracy ofMMMethods (In \%) } \\
\hline $\begin{array}{c}\text { Sampling Interval (in } \\
\text { sec.) }\end{array}$ & ST-MM & FIS-MM & Statistics & Grid ST \\
\hline 20 & 0.90 & 0.94 & 0.92 & 0.91 \\
\hline 40 & 0.92 & 0.925 & 0.925 & 0.915 \\
\hline 60 & 0.91 & 0.91 & 0.89 & 0.90 \\
\hline 80 & 0.89 & 0.90 & 0.87 & 0.88 \\
\hline 100 & 0.87 & 0.89 & 0.85 & 0.86 \\
\hline
\end{tabular}

shows the lowest matching accuracy and Fuzzy logic dependent IntelliAssistant MM displays better accuracyand highly insensitive to sampling interval variance. The effects of an outlier to performance in terms of recall and precision for SPMC based Intelliassistant with previous next location prediction method i.e. TM Based and Ignorant Based route recommendation are represented in Figure 12.

\section{CONCLUSION}

The IntelliAssistant MM algorithm involves isolation of every sampling point to the appropriate location across the real road to recover the actual route covered by the individual. On the other hand implementation and evaluation of the SPMC have been done with the aim is to adapt the route to user preferences. Experiments reveal that the MM algorithm integrated to IntelliAssistant focused on Fuzzy logic achieves substantial efficiency enhancements in runtime and precision relative to previous policies such as GridST, Statistics, and LBS ST-Matching. Fuzzy MM algorithm dependent reasoning is a reasonable alternative for large trajectory collections of specimens. As a future work we will use big datasets for real-world fuzzy log dependent MM to be integrate in IntelliAssistant that would involve tremendous effort in data cleaning. The avenue for potential work is, thus, to simplify data cleaning procedures especially for heterogeneous type data sources. 
Figure 12. Precision and recall comparison of SPMC based IntelliAssistant with different next location prediction based route recommendation methods
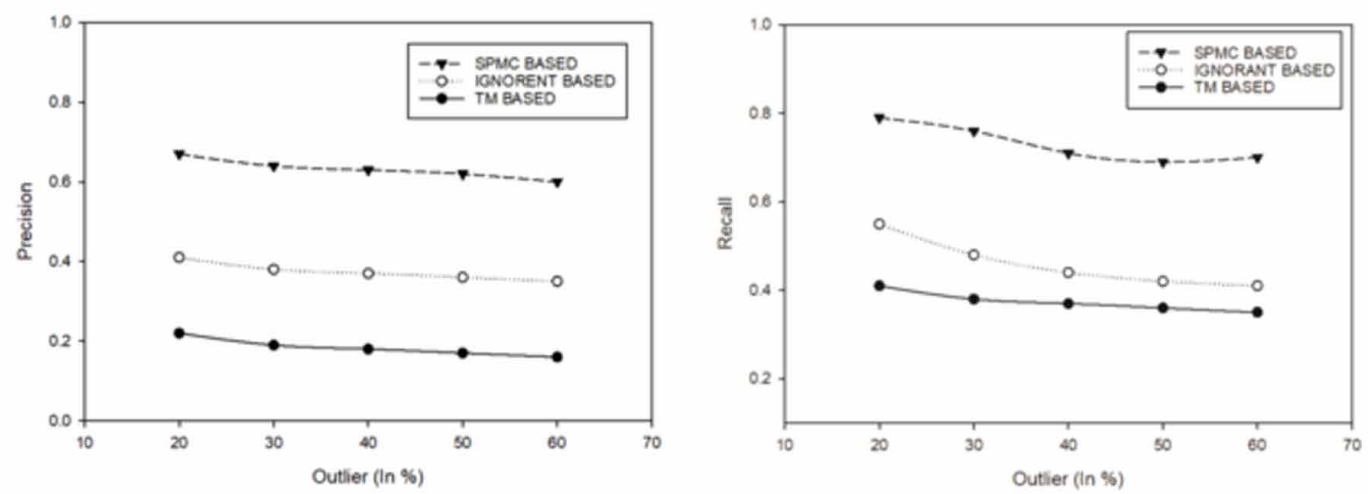


\section{REFERENCES}

Akin, D., \& Alasalvar, S. (2016). Estimate Urban Growth and Expansion by Modeling Urban Spatial Structure Using Hierarchical Cluster Analyses of Interzonal Travel Data. International Journal of System Dynamics Applications, 5(4), 16-41.

Algizawy, E., Ogawa, T., \& El-Mahdy, A. (2017). Real-time large-scale MM using mobile phone data. ACM Transactions on Knowledge Discovery from Data, 11, 52.

American Psychiatric Association. (2013). Diagnostic and Statistical Manual of Mental Disorders (DSM-5) (5th ed.). American Psychiatric Publishing.

Bajracharya, A. (2016). Public Transportation and Private Car: A System Dynamics Approach in Understanding the Mode Choice. International Journal of System Dynamics Applications, 5(2), 1-18.

Battini, D., Faccio, M., Persona, A., \& Sgarbossa, F. (2013). Modelling the Growing Process of Integrated Healthcare Supply Networks. International Journal of System Dynamics Applications, 2(1), 1-13. doi:10.4018/ ijsda.2013010101

Carmien, S., Dawe, M., Fischer, G., Gorman, A., Kintsch, A., \& Sullivan, J. F. Jr. (2005). Socio-technical environments supporting people with cognitive disabilities using public transportation. ACM Transactions on Computer-Human Interaction, 2005(12), 233-262. doi:10.1145/1067860.1067865

Chandio, A. A., Tziritas, N., Zhang, F., \& Xu, C.-Z. (2015). An approach for map-matching strategy of GPStrajectories based on the locality of road networks. In IOV 2015. LNCS (Vol. 9502, pp. 234-246). Springer.

Cho, Y., \& Choi, H. (2014). Accuracy enhancement of position estimation using adaptive Kalman filter and MM. Int. J. Control Automat., 7(7), 167-178.

Dawe, M. (2006). Desperately seeking simplicity: How young adults with cognitive disabilities and their families adopt assistive technologies. In Proceedings of the SIGCHI Conference on Human Factors in Computing Systems. ACM. doi:10.1145/1124772.1124943

Elharakany, R. A., Moscardini, A., Khalifa, N. M., \& Elghany, M. M. (2018). Modelling the Effect on Quality of Information and Communications Technology (ICT) facilities in Higher Education: Case Study-Egyptian Universities. International Journal of System Dynamics Applications, 7(3), 1-30. doi:10.4018/IJSDA.2018070101

Fournier-Viger, P. (2017). SPMF - open-source data mining mining library. Academic Press.

Georgiou, C., Anderson, S., \& Dodd, T. (2017). Constructing informative bayesian map priors: A multi-objective optimisation approach applied to indoor occupancy grid mapping. The International Journal of Robotics Research, 36(3), 274-291.

Gong, Y.-J., Chen, E., Zhang, X., Ni, L. M., \& Zhang, J. (2017, February). AntMapper: An ant colony-based MM approach for trajectory-based applications. IEEE Transactions on Intelligent Transportation Systems, 19(2), 390-401.

Gupta, A., \& Shanker, U. (2020a). OMCPR: Optimal Mobility Aware Cache Data Pre-fetching and Replacement Policy Using Spatial K-Anonymity for LBS. Wireless Personal Communications, 114(2), 949-973. doi:10.1007/ s11277-020-07402-2

Gupta, A. K. (2020). Spam Mail Filtering Using Data Mining Approach: A comparative performance analysis. In S. Shanker, U., Pandey (Ed.), Handling Priority Inversion in Time-Constrained Distributed Databases (pp. 253-282). Hershey, PA: IGI Global, 2020. https://doi.org/10.4018/978-1-7998-2491-6.ch015

Gupta, A. K., \& Prakash, S. (2018). Secure Communication in Cluster-Based Ad Hoc Networks: A Review. In D. Lobiyal, V. Mansotra, \& U. Singh (Eds.), Next-Generation Networks. Advances in Intelligent Systems and Computing (Vol. 638). Springer., doi:10.1007/978-981-10-6005-2_54

Gupta, A. K., \& Shanker, U. (2017). Modified Predicted Region based Cache Replacement Policy for Location Dependent Data in Mobile Environment. Sixth Intern. Conf. on Smart Compu. \& Comm. 2017, 917-924.

Gupta, A. K., \& Shanker, U. (2018). CELPB: A cache invalidation policy for location dependent data in mobile environment. ACM International Conference Proceeding Series. doi: doi:10.1145/3216122.3216147 
Gupta, A. K., \& Shanker, U. (2019). SPMC-PRRP: A Predicted Region Based Cache Replacement Policy. In Lecture Notes in Networks and Systems (Vol. 39). https://doi.org/doi:10.1007/978-981-13-0277-0_26

Gupta, A. K., \& Shanker, U. (2020). Study of fuzzy logic and particle swarm methods in map matching algorithm, SN Appl. Sci. 2, 608 (2020). https://doi.org/10.1007/s42452-020-2431-y

Gupta, A. K., \& Shanker, U. (2020). MAD-RAPPEL: Mobility Aware Data Replacement \&Prefetching Policy Enrooted LBS. Journal Journal of King Saud University - Computer and Information Sciences.

Gupta, A. K., \& Shanker, U. (2020). A Literature Review of Location-Aware Computing Policies: Taxonomy and Empirical Analysis in Mobile Environment. [IJMHCI]. International Journal of Mobile Human Computer Interaction, 12(3), 21-45. doi:10.4018/IJMHCI.2020070102

Gupta, A. K., \& Shanker, U. (2021). An Efficient Markov Chain Model Development based Prefetching in Location-Based Services. In P. Saravanan, \& S. Balasundaram (Ed.), Privacy and Security Challenges in Location Aware Computing (pp. 109-125). IGI Global. http://doi:10.4018/978-1-7998-7756-1.ch005

Gupta, A. K., \& Shanker, U. (2021). Mobility-Aware Prefetching and Replacement Scheme for Location-Based Services: MOPAR. In P. Saravanan \& S. Balasundaram (Eds.), Privacy and Security Challenges in Location Aware Computing (pp. 26-51). IGI Global., http://doi:10.4018/978-1-7998-7756-1.ch002

Gupta, A. K., \& Shanker, U. (2018). Location Dependent Information System's Queries for Mobile Environment. 23rd International Conference on Database Systems for Advanced Applications (DASFAA) International Workshops, 1-9.

Gupta, Ajay Kr, \& Shanker, U. (2020). Some Issues for Location Dependent Information System Query in Mobile Environment. 29th ACM International Conference on Information and Knowledge Management (CIKM '20), 4. https://doi.org/. https://doi.org/10.1145/3340531.3418504

Hart, P. E., Nilsson, N. J., \& Raphael, B. (1968). A formal basis for the heuristic determination of minimum cost paths. IEEE Transactions on Systems Science and Cybernetics, 4(2), 100-107.

Hsueh, Y. L., \& Chen, H. C. (2018). MM for low-sampling-rate GPS trajectories by exploring real-time moving directions. Inf. Sci., 433, 55-69.

Hu, J., Cao, W., Luo, J., \& Yu, X. (2009). Dynamic modeling of urban population travel behavior based on data fusion of mobile phone positioning data and FCD. Proceedings of the 2009 17th International Conference on Geoinformatics, 1-5.

Karich, P., Schroder, S. (2017). GraphHopper Directions API with Route Optimization. Academic Press.

Kizito, A., \& Semwanga, A. R. (2020). Modeling the Complexity of Road Accidents Prevention: A System Dynamics Approach. International Journal of System Dynamics Applications, 9(2), 24-41.

Knapen, L., Bellemans, T., Janssens, D., \& Wets, G. (2018). Likelihood-based offline MM of GPS recordings using global trace information. Transportation Research Part C, Emerging Technologies, 93, 13-35.

Liang, B., Wang, T., Chen, W., Li, H., Lei, K., \& Li, S. (2016). Online learning for accurate real-time MM. Proc. 20th Pacific-Asia Conf. Adv. Knowl. Discovery Data Mining, 9652, 67-78.

Liu, A. L., Hile, H., Borriello, G., Kautz, H., Brown, P. A., Harniss, M., \& Johnson, K. (2009). Informing the Design of an Automated Wayfinding System for Individuals with Cognitive Impairments. Proceedings of the 3rd International Conference on Pervasive Computing Technologies for Healthcare, 9, 8. doi:10.4108/ICST. PERVASIVEHEALTH2009.6018

Liu, X., Liu, K., Li, M., \& Lu, F. (2017, May). A ST-CRF map-matching method for low-frequency floating car data. IEEE Transactions on Intelligent Transportation Systems, 18(5), 1241-1254.

Lou, Y., Zhang, C., Zheng, Y., Xie, X., Wang, W., \& Huang, Y. (2009). Map-matching for low-sampling-rate GPS trajectories. In Proceedings of 17th ACM SIGSPATIAL International Symposium on Advances in Geographic Information Systems. ACM.

Millard-Ball, A., Hampshire, R. C., \& Weinberger, R. R. (2019). Map-matching poor-quality GPS data in urban environments: The pgMapMatch package. Transportation Planning and Technology, 42, 539-553. 
Mohamed, R., Aly, H., \& Youssef, M. (2017, April). Accurate real-time MM for challenging environments. IEEE Transactions on Intelligent Transportation Systems, 18(4), 847-857.

Oyama, Y., \& Hato, E. (2017). A discounted recursive logit model for dynamic gridlock network analysis. Transportation Research Part C, Emerging Technologies, 85, 509-527. https://doi. org/10.1016/j.trc.2017.10.001

Oyama, Y., \& Hato, E. (2018). Link-based measurement model to estimate route choice parameters in urban pedestrian networks. Transportation Research Part C, Emerging Technologies, 93, 62-78. https://doi. org/10.1016/j.trc.2018.05.013

Qin, G., Huang, Z., Xiang, Y., \& Sun, J. (2019). ProbDetect: A choice probability-based taxi trip anomaly detection model considering traffic variability. Transportation Research Part C, Emerging Technologies, 98, 221-238. https://doi.org/10.1016/j.trc.2018.11.016

Qinglin, T., Zoran, S., \& Kevin, I. W. (2015). A Hybrid Indoor Localization and Navigation System with MM for Pedestrians Using Smartphones. Sensors (Basel), 15, 30759-30783.

Quddus, M. A., Noland, R. B., \& Ochieng, W. Y. (2006). A high accuracy fuzzy logic based MM algorithm for road transport. Journal of Intelligent Transport Systems, 10(3), 103-115.

Rahmani, M., Koutsopoulos, H. N., \& Jenelius, E. (2017). Travel time estimation from sparse floating car data with consistent path inference: A fixed point approach. Transportation Research Part C, Emerging Technologies, 85, 628-643. https://doi.org/10.1016/j.trc.2017.10.012

Ramos, J., Anacleto, R., Novais, P., Figueiredo, L., Almeida, A., \& Neves, J. (2013). Geo-localization System for People with Cognitive Disabilities. In Trends in Practical Applications of Agents and Multiagent Systems (Vol. 221, pp. 59-66). Springer.

Rappos, E., Robert, S., \& Cudré-Mauroux, P. (2018). A force-directed approach for offline GPS trajectory MM. Proceedings of the 26th ACM Sigspatial International Conference on Advances in Geographic Information Systems, 319-328.

Ren, M., \& Karimi, H. A. (2009). A hidden Markov model-based map-matching algorithm for wheelchair navigation. Journal of Navigation, 62, 383-395.

Sadilek, A., \& Kautz, H. (2010). Recognizing Multi-Agent Activities from GPS Data. Artificial Intelligence, 39, 1134-1139.

Schuessler, N., Axhausen, K., \& Zurich, E. (2009). Map-matching of GPS traces on high resolution navigation networks using the multiple hypothesis technique (Vol. 1). MHT.

Schwertfeger, S., \& Yu, T. (2016). Matching paths in topological maps. In 9th Symposium on Intelligent Autonomous Vehicles (IAV). IFAC.

Sharath, M. N., Velaga, N. R., \& Quddus, M. A. (2019). A dynamic two-dimensional (D2D) weight-based mapmatching algorithm. Transportation Research Part C, Emerging Technologies, 98, 409-432.

Spichkova, M., \& Hamilton, M. (2016). Dynamic Decision Making System for Public Transport Routes. [IJSDA]. International Journal of System Dynamics Applications.

Spichkova, M., \& Hamilton, M. (2016). Dynamic Decision Making System for Public Transport Routes. International Journal of System Dynamics Applications, 5(3), 47-70.

Tang, K., Chen, S., Liu, Z., \& Khattak, A. J. (2018). A tensor-based Bayesian probabilistic model for citywide personalized travel time estimation. Transportation Research Part C, Emerging Technologies, 90, 260-280. https://doi.org/10.1016/j.trc.2018.03.004

Wang, H., Li, J., Hou, Z., Fang, R., Mei, W., \& Huang, J. (2017). Research on parallelized real-time MM algorithm for massive GPS data. Cluster Computing, 20, 1123-1134.

Wei, H., Wang, Y., Forman, G., \& Zhu, Y. (2013). MM by Fréchet Distance and Global Weight Optimization. Technical Paper. Department of Computer Science and Engineering.

Wolfson, O., Sistla, A. P., Chamberlain, S., \& Yesha, Y. (1999). Updating and querying databases that track mobile units. Distributed and Parallel Databases, 7(3), 257-387. 
Yang, C., \& Gidófalvi, G. (2018). Fast MM, an algorithm integrating hidden Markov model with precomputation. International Journal of Geographical Information Science, 32(3), 547-570.

Yin, Y., Shah, R. R., Wang, G., \& Zimmermann, R. (2018). Feature-based MM for Low-Sampling-Rate GPS Trajectories. Trans. Spat. Algorithms Syst., 4, 1-24.

Yin, Y., Shah, R. R., \& Zimmermann, R. (2016). A general feature-based MM framework with trajectory simplification. Proc. ACM SIGSPATIAL Int. Workshop Geostreaming, 7.

Yuan, J., Zheng, Y., Zhang, C., Xie, X., \& Sun, G. Z. (2010). An interactive-voting based MM algorithm. Proceedings of the 2010 Eleventh International Conference on Mobile Data Management, 43-52.

Zhao, S., Hrbek, S., Lu, M., \& Akos, D. (2014). Deep Integration of GPSINS Based on a Software Defined Receiver - Implementation and Test Results. In 27th International Technical Meeting of the Satellite Division of the Institute of Navigation. ION GNSS.

Zhou, Y. (2013). A PSO-Inspired Multi-Robot Map Exploration Algorithm Using Frontier-Based Strategy. International Journal of System Dynamics Applications, 2(2), 1-13.

Zhu, L., Holden, J. R., \& Gonder, J. D. (2017). Trajectory Segmentation Map-Matching Approach for LargeScale, High-Resolution GPS Data. Transportation Research Record: Journal of the Transportation Research Board, (2645), 67-75.

Ajay Kr. Gupta is presently a Ph.D Research Scholar in the Department of Computer Sc. \& Engineering of M. M. M. University of Technology, Gorakhpur 273010. His current research areas are Spatio-Temporal Database, Location Dependent Database, and Mobile Distributed Database.

Udai Shanker $(P h D)$ is presently Professor in the Department of Computer Sc. \& Engineering of M. M. M. University of Technology, Gorakhpur-273010. He is credited with PhD from Indian Institute of Technology Roorkee and is recipient of awards from Institution of Engineers (India), Calcutta twice for his technical papers. He is authors of 80 research papers, which have been published in various National \& International Journals/Conferences. He is reviewer of many International Conferences/Journals and also Editorial Board Member of 9 International Journals. 